\title{
Über die periphere Regulierung der Blutzirkulation.
}

Von

w. R. Hess.

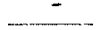

(Mit 12 Textfiguren.)

I.

Eine Eigenschaft ist es, welche die Funktionen des lebenden Blutgefässsystemes weit über. die Leistungen eines jeden toten Leitungssystemes erhebt; es ist dies die Anpassungsfähigkeit an die wechselnden Ansprüche des Versorgungsgebietes in bezug auf die zuzuführende Blutmenge.

Einen wesentlichen Anteil bei dieser Anpassung übernimmt das Herz durch eine verschiedene Einstellung seiner Druckleistung und seïnes sekundlichen Fördervolumens. Die Stellung des Herzens als zirkulatorisches Zentrum bringt es jedoch mit sich, dass es nicht den speziellen Bedürfnissen einzelner Regionen Rechnung tragen kann, sondern stets nur dem durchschnittlichen Bedarf aller Organe zusammen. Ein soleher Durchschnittsbedarf kann aber in sehr verschiedener Weise zustande kommen, d. h. unter wechselnder Beteiligung der einzelnen Regionen. Unabhängig voneinander können sich lokale Ansprüche ändern, hier steigen, dort fallen, sich ganz oder teilweise kompensierend. An der vom Herzen gelieferten Sekundenmenge braucht in solchen Fällen nichts oder nur wenig geändert zu werden. Eine einschneidende Veränderung muss dagegen Platz greifen in bezug auf die Aufteilung des vom Zentrum gelieferten Blutstromes auf die einzelnen Regionen des Blutgefässnetzes. D i e Veränderung sozu besorgenund immerfortabzuändern, dassallerortsden lokalen Zirkulationsansprüchen und deren Schwankungen entsprochen.wird, das ist die Aufgabe der peripheren Regulation der Blut/irkula- 
tion. Ihr Wirkungsfeld erstreckt sich von derjenigen Stelle an, wo sich die einzeluen grossen Stromgebiete von der Aorta abtrennen bis hinaus in die äusserste Peripherie, wo es gilt, jedem kleinsten Gewebebezirk diejenige Blutmenge zuzuweisen, deren es zur Aufrechterhaltung seiner Leistungen bedarf.

Wodurch diese periphere Regulierung bewirkt wird, darüber sind wir nicht im Zweifel; sie gesehieht durch den aktiven Einfluss der Gefüssmuskulatur. Offenkundig ist es auch, dass hierbei die Gefässnerven und Gefässnervenzentren eine koordinierende Funktion ausüben.

Wi e aber der spezielle Mechanismus gestaltet ist, welcher die Steuerung des Blutstromes besorgt, darüber sind wir noch sehr mangelhaft orientiert. Wie weit hierüber die Kenntnisse heute reichen, soll nun vorerst festgestellt werden.

Wir beginnen damit, dass wir kurz an einige Beobachtungen erinnern, durch welche die tatsächliche Existenz einer peripheren Kreislaufregulierung nachgewiesen ist.

Fine solche Beobachtung ist die Konstatierung von $\mathrm{Ch}$ auveau und Kaufmann ${ }^{1}$ ). Diese Autoren haben das aus dem Musculus levator labii superior des Pferdes ausfliessende Venenblut in der Ruhe , und während der Tätigkeit des Muskels gemessen und dabei gefunden, dass der Blutabfluss - somit auch der Blutzufluss während der Betätigung des betreffenden Muskels das Vier-bis Sechsfache des Ruhewertes beträgt. - Die Arbeitsleistung des auf seine Durcbblutungsgrösse untersuchten Muskels geschah in diesen Experimenten unter dem Einfluss der $\mathrm{ph}$ y s i o log i s chen Innervation.

Dass auch bei Muskelaktion, herbeigeführt durch $k \ddot{u} n s t l i c h e$ Reizung der Muskelnerven, dieselbe Erscheinung eines Anschwellens der Durchblutung zustande kommt, zeigte zuerst Gaskel1²) (loc. cit. Tafel IX Fig. 5) durch seine Versuche am Musculus gracilis des Hundes.

Ganz analoge Verhältnisse wurden in bezug auf die Durchblutung von Drüsen gefunden. Es geht dies aus den Versuchen von Bar$\operatorname{crof} \mathrm{t}^{3}$ ) und anderen Autoren hervor, dass nämlich zum Beispiel die vermehrte Sekretion der Glandula submaxillaris (der Katze) bei

1) Chanveau pund Kaufmann, Arch. de Physiol. 1892 p. 279.

2) The Journ. of Physiol. vol. 1 p. 276.

3) The Journ. of Physiol. vol. 36 p. liii. 
Chordareizung begleitet ist von einer starken Steigerung des Blutstromes, der sich aus den Drüsenvenen ergiesst, und zwar geht, nach der Analyse der Blutproben zu schliessen, die Erhöhung des minutlichen Durchflussvolumens annähernd der Vermehrung des Stoffumsatzes parallel.

Weiterhin sind hier die Untersuchungen von "Landergreen und Tigerstedt ${ }^{1}$ ) über die Blutversorgung der Niere bei Injektion von Kochsalz, Nitraten und Koffein anzuführen. Auch hier wieder dieselbe Erscheinung: Steigerung der Blutversorgung bei Zunahme der Tätigkeit eines Organes als Ausdruck der Steigerung seines Blutbedarfes.

Soviel zur Frage der Existenz einer peripheren Durchblutungsregulierung.

Zur Orientierung über unsere heutigen Kenutnisse der Wirkungsweise des steuernden Mechanismus zitiere ich die Ausführungen, welche B a y li ss ${ }^{2}$ ) in einer zusammenfassenden Darstellung über diesen Gegenstand gegeben hat:

„Wenn ein Organ infolge vermehrter Tätigkeit eine grössere Blutversorgung erfordert, gibt es mehrere Wege, auf welchen hierfür gesorgt werden kann. In manchen Organen ist eine dieser Methoden die hauptsächlichste, in anderen Organe jene Methode. Manchmal wirken mehr als eine gleichzeitig.

Diese verschiedenen Methoden sind:

1. Erregung der gefässerweiternden Nerven vom Zentralnervensystem aus.

2. Hemmung der zentralen tonischen Erregung der Vasokonstriktoren. Bei typischen Reflęxen sind diese beiden Wirkungen gleichzeitig vorhanden.

3. Vasokonstriktion in anderen Organen, durch welche der allgemeine arterielle Druck erhöht wird. Bei den Lovèn-Reflexen werden die drei ersten Wirkungen gemeinschaftlich hervorgerufen.

4. Durch die Produktion infolge Stoffwechseltätigkeit des Organs gewisser chemischer Substanzen, welche auf die Blutgefässe direkt wirken, indem sie Erweiterung hervorrufen. Diese Körper können derartig wie Kohlensäure sein, für welche, wie es scheint, die Arteriolen ganz allgemein empfindlich sind. Aber wegen ihrer grösseren

1) E. Landergreen und $R$. Tigerstedt, Die Blutzufuhr zur Niere. Skandinav. Arch. f. Physiol. Bd. 4 S. 241. 1893.

2) Ergebn. f. Physiol. Bd. 5 S. 346 u. ff. 
Konzentration am Entstehungsorte wirken sie hauptsächlich daselbst. Anderseits können auch Körper erzeugt werden, für welche die Blutgefässe eines besonderen Organes spezifisch empfindlich sind, wie beispielsweise das Pankreas für Substanzen, welche in der Schleimhaut des Duodenums erzeugt werden, und möglicherweise der Darm selbst gegen seine eigenen Produkte.

Wir sehen also, dass reichlich Vorkehrungen getroffen sind für die Regulation der Blutversorgung zu den Organen je nach deren erforderlichen Bedürfnissen."

Die Orientierung, welche wir durch diese Zusammenfassung erhalten, beschränkt sich, wie wir sehen, im wesentlichen auf die Registrierung einer Anzahl Faktoren! Damit sind wir aber noch weit davon entfernt, uns ein einigermaassen klares Bild davon machen zu können, wie diese verschiedenartigen "Vorkehrungen" zu einem wohlgeordneten Mechanismus koordiniert sind.

Eine wesentliche Ergänzung erfährt unser Verständnis für die Frage, wenn wir dem Gedankengang folgen, den Roux ${ }^{1}$ ) über die Anpassung der Gefässweiten entwickelt hat:

"Die Anpassungsfähigkeit der Gefässweite ist eine zweifache; eine ,rein funktionelle, zu raschem Wechsel geeignete und eine danerndere, wirklich gestaltliche.

Die erstere wird durch Nerven vermittelt und beschränkt sich ihrer Art nach rein auf die Dimensionen der Weite ohne Änderung der Länge.

Diese Regulation ist durch physiologische und klinische Beobachtungen sichergestellt. Dass sie für die Gehirnarterien feble, halte ich für ausgeschlossen. Sie veranlasst Erweiterung der Gefässe durch nervös vermittelte Änderung des Tonus der Ringmuskeln, welche dabei wohl durch den Blutdruck passiv verlängert werden.

Sie bewirkt normalerweise die funktionelle Hyperämie, also die Vermehrung`der Blutzufuhr bei der Funktion der Organe, sicher wenigstens der aktiv tätigen Organe der Muskeln, Drüsen und Nervenzentren. Ihre Wirkung ist so gross, dass sie den Blutgehalt eines Organes in wenigen Minuten fast verdoppeln kann.

Sie kann offenbar in genau dem Verbrauche des ernährten Parenchyms entsprechendem Maassegeleitet

1) Vgl. Oppel, Uber die gestaltliche Anpassung der Blutgefässe. Vortr. u. Aufs. über Entwicklungsmech. H. 10 (mit Orig.-Beig. v. W, Roux). 
werden, und diese Leitang muss vom verbrauchenden Parenchym selber ausgehen. Von einem anderen Ort aus ist diese Regulation in genügend feiner Weise nicht möglich, wenn wir auch wissen, dass bei Änderung des Bedarfes ganzer Organe und noch grösserer Bezirke die einheitliche Vermittlung der nervösen Gefässzentren in Anspruch genommen wird. Anderseits ist anzunebmen, dass das Gehirn bei der von ihm selber veranlassten Tätigkeit der Organe, zum Beispiel der Muskeln, Augen, wohl auch gleich die zugehörigen Blutbahnen, Arterien und Venen erweitern lässt, so dass von dem tätigen Organ nicht erst an das Gefässzentrum telegraphiert und gleichsam an Nahrung gebettelt werden muss wie von einem unzureichend bezahlten Diätar. Aber diese vom Zentrum ausgehende Regulation kann unmöglich genau genug der verschieden starken Tätigkeit der Teile eines Muskels entsprechend lokalisiert und abgestuft werden. Dies Geschehen ist noch weniger möglich für die überhaupt nicht vom Gehirn ausgehende Tatigkeit der Verdauungsorgane; und sie ist ganz unmöglich für das vom Nervenzentrum unabhängige Wachstum der Organe und der Tumoren. Da aber auch für dieses Wachstum zureichende Regulation existiert, muss also auch eine direkte, vom Parenchym aus vermittelte Regulation der Gefässweite existieren. Diese Regulation muss bereits mit den ersten Blutgefässen im Tierreich entstanden sein; und seitdem muss jede neue Arterie, eventuell auch Vene und Kapillare sowie das zugehörige Parenchym, sofort mit Anschluss an den Regulationsmechanismus der vorher vorhandenen Gefässe versehen worden sein. Bei gesteigertem Verbrauch wird von der Verbrauchsstelle aus zentral gegen das Herz hin in den Nerven der Arterien der Reiz zur Erweiterung fortgeleitet; dies un so weiter zentral, je stärker die Erregung an der einzelnen Stelle ist, und je mehr Kapillaren zugleich vom Parenchym aus erweitert bzw. neugebildet sind."

Es genügt ein Hinweis darauf, dass sich die Roux'schen, auf theoretischer Grundlage entwickelten Vorstellungen den von B ayliss zusammengefassten Resultaten der experimentellen Forschung zum Teil sehr gut und ohne Zwang anpassen. Hervorgehoben seien zum Beispiel die Ausführungen, welche Roux über die Notwendigkeit einer Kombination der vom Zentrum ausgehenden Regulierungsimpulse mit einer Beeinflussung der Gefässe vom Parenchym aus gibt. Diese Forderung finden wir tatsächlich erfüllt im Sinne von Ziffer 1 und 4 der Bayliss' schen Ausführungen. (Vgl. S. 441.) Pflüger's Archiv für Physiologie. Bd. 168. 
Es möge hier noch die Gelegenheit wahrgenommen werden, auf die Arbeiten von $\mathrm{Thoma}^{1}$ ) und $\mathrm{Fuchs}^{2}$ ) hinzuweisen, welcheAutoren sich eingehend mit der Frage der Querschnittsentwicklung der'Blutgefässe beschäftigt haben. Dabei haben sie aber die Wachstumsentwicklung und nicht, wie wir, die regulatorische $Q u e r-$ schnittsveränderung im Auge, deren Zweck die zirkulatorische Anpassung an den mit der Funktionsintensität wechselnden Blutbedarf der Organe ist. Da zwischen den 'zwei Einstellmechanismen - wie schon Roux betont - wohl ein Zusammenhang besteht, sei immerhin das Wesentliche aus den zitierten Arbeiten Tho m a's erwähnt. Nach diesem Autor ist der maassgebende Faktor, welcher den Querschnitt eines Gefässes bestimmt und reguliert, die Strömungsg eschwindigkeit. Überschreitet sie einen bestimmten Betrag, so löst sie Querschnittszunahme aus; unterschreitet sie eben diese Grenze, so wird Reduktion des Querschnittes eingeleitet. - Wie sich der Einfluss der Geschwindigkeit auf die Gefässwand überträgt, darüber äussert sich Th o m a nicht bestimmt. Dagegen findet er in der von ihm dargelegten Berechnung Beweiskraft für die Richtigkeit seiner Theorie. -

Die Berechnungen basieren jedoch, so viel können wir nach eigenen Untersuchungen mit Bestimmtheit aussagen, auf einer ârchaus irrtumlichen Interpretation der Beobachtungen von Demning und Watson ${ }^{3}$ ) über das Strömen des Blutes in engen Röhren.

Die darauf begründeten Ausführungen Thoma's lassen sich deshalb unter keinen Umständen aufrechterbalten.

Soviel zum heutigen Stand unserer Kenntnisse über den Mechanismus der peripheren Kreislaufregulierung.

Wir gehen zu unseren eigenen Untersuchungen über:

In den Vorgängen der peripheren Zirkulationsregulierung haben wir den Effekt einer gemeinschaftlichen Betätigung der Gefässmuskulatur und der Gefässnerven mit ihren Zentren vor uns.

1) Thoma, Histogenese und Histomechanik des Gefässsystems. Ferd. Enke, Stuttgart 1893. - Th oma, Die Viskosität des Blutes und seine Strömung. im Arteriensystem. Deutsch. Arch. f. klin. Med. Bd. 99 H. 5 und 6. 1910.

2) R. Fuchs, Zur Physiologie und Wachstumsmechanik des Blutgefässsystems. Zeitschr. f. allgem. Physiol. Bd. 2 S. 15. 1903.

3) Vgl. Thoma, Deutsch. Arch. f. klin. Med. Bd. 99 S. 581 H. 5 und 6. W. R. Hess, Gehorcht das Blut dem allgemeinen Strömungsgesetz von Flüssigkeiten? Pflüger's Arch. Bd. 162 S. 203. 
Dem Muskelapparat fällt die Aufgabe zu, durch einen oder verschiedene mechanische Akte die Strömungsbedingungen zu verändern; dies geschieht auf dem Wege der Widerstandsänderung, herbeigeführt durch aktiven Querschnittswechsel. Der Gefässnervenapparat besorgt die Aufgabe, die über die gesamte Peripherie zerstreute Muskulatur derart zu einem organisehen System zusammenzufassen, dass sie in Form assoziierter und dosierter Akte wirksam wird; in Form von Akten, deren Einfluss auf die Blutströmung im Einklang mit Änderungen im Blutbedarf des versorgten Gewebes steht.

Díesen Verhältnissen entsprechend bieten sich dem Studium der Kreislaufregulierung zwei natürlich getrennte Untersuchungsgebiete dar.

Das erste umfasst die Untersuchung der mechanischen Vorgänge, durch welche die Kreislaufregulierung in das Strömen des Blutes eingreift.

Das zweite betrifft die koordinatorischen Leistungen des G e fä s snervenapparates.

Das Studium der Literatur lässt keinen $Z$ weifel darüber bestehen, dass in der bisherigen Forschung die zweite Frage ein gewaltiges Übergewicht erhalten hat. Es ist möglich, dass dies so geschehen, weil die Gefässmuskelwirkung für vollständig abgeklärt gehalten wurde. Tatsache ist jedoch, dass dieses Kapitel noch manch unbeantwortete Frage birgt, deren Lösung für das Verständnis des zweiten Abschnittes nicht nur nützlich, sondern notwendig ist.

Denn der Finfluss der Gefässmuskelaktion auf den Abiauf der Zirkulation ist der mechanische Faktor, auf dem sichdieganzeperiphere Kreislaufregulierung aufbaut. Die genaue Kenntnis dieser mechanischen Beziehung muss es auch sein, welche uns die Grundlage zu bilden hat für Erforschung und Verständnis der Vorgänge, durch welche der Gefässmuskel seine stromregulierenden Funktionen erledigt.

\section{Die Dynamik der Gefässmuskulatur.}

a) Die dynamischen Beziehungen zwischen der Muskulatur paralle1 geschalteter Gefäissstrecken.

In den eben ausgeführten Sätzen liegt die Begründung, weshalb wir als erstes die "Dynamik der. Gefässmuskulatur" behandeln. Es 
ist dabei unser Ziel, an Stelle der bisherigen sehr summarischen Vorstellungen exaktere, wenn möglich quantitativ präzisierte zu setzen. Die Vorstellungen, mit denen man heute zu rechnen gewohnt ist, lassen sich durch folgende Sätze umschreiben:

1. Erweiterung der Gefässe eines umgrenzten Zirkulationsgebietes führt zu einer Steigerung dessen Durchblutung.

2. Diese lokale Steigerung ist begleitet von einer Stromverminderung in anderen Stromgebieten infolge der Ablenkung. des Blutstromes nach den Erweiterungsgebieten.

3. Als indirekte Konsequenz ist die Reaktion ferner begleitet von einer zentralen Blutdrucksenkung (soweit wir ausschliesslich Einfluss der Peripherie auf die Gestaltung des Blutdruckes im Ange behalten).

4. Gefässverengerung bewirkt in jeder Beziehung den umgekehrten Effekt, nämlich auf direktem Wege: Verminderung der Durchblutung im Verengerungsgebiet; indirekt: Erhöhung der Durchblutung anderer Stromgebiete und zentrale Blutdrucksteigerung.

Den Inhalt dieser allgemein bekannten hämodynamischen Regeln gilt es nun schärfer zu differenzieren.

Einen Ansatz in dieser Richtung hat $\mathrm{Fuchs}^{1}$ ) gemaeht in Form einer mechanischen Analyse der Gefässkontraktion. Diese Analyse bezieht sich jedoch lediglich' auf die Mechanik des Kontraktionsprozesses ohne Berücksichtigung der zirkulatorischen Konsequenzen. Dieseletzteren sind es aber, welchefürdie Kreislaufregulierung das entscheidende Moment darstellen, und die wir deshalb studieren.

Eine erste Frage, welche zu beantworten ist, lautet dahin, ob ein Eingreifen der Gefässwand in die Blutzirkulation dadurch zustande kommt, dass durch sie das Blut einen Strömungsantrieb erbält. Bei einer solchen Funktion der Gefässmuskulatur wäre diese also in Parallele zu setzen mit der Herzmuskulatur, so dass man von der Summe aller Gefässmuskeln als sogenanntem peripheren Herzen sprechen könnte. Es liegt auf der Hand, dass ein aktives Förderungsvermögen der Gefässwände gerade bei der Kreislaufregulierung ausgiebige Gelegenheit zur Betätigung finden würde und demgemäss die Regulationsmechanik tiefgreifend beeinflussen müsste.

1) Fuchs; 1. c. S. 444. 
Um dieser Frage eine eingehende Behandlung zuteil werden zu lassen, habe ich sie aus dieser Arbeit ausgesondert und getrennt behandelt ${ }^{1}$ ). Es genüge, hier das Resultat anzuführen, zu welchem wir gelangt sind. Es lautet dahin, dass wir den Arterien die Fähigkeit, den Blutstrom aktiv zu fördern, absprechen, also die Existenz des sogenannten "peripheren Herzens" verneinen müssen.

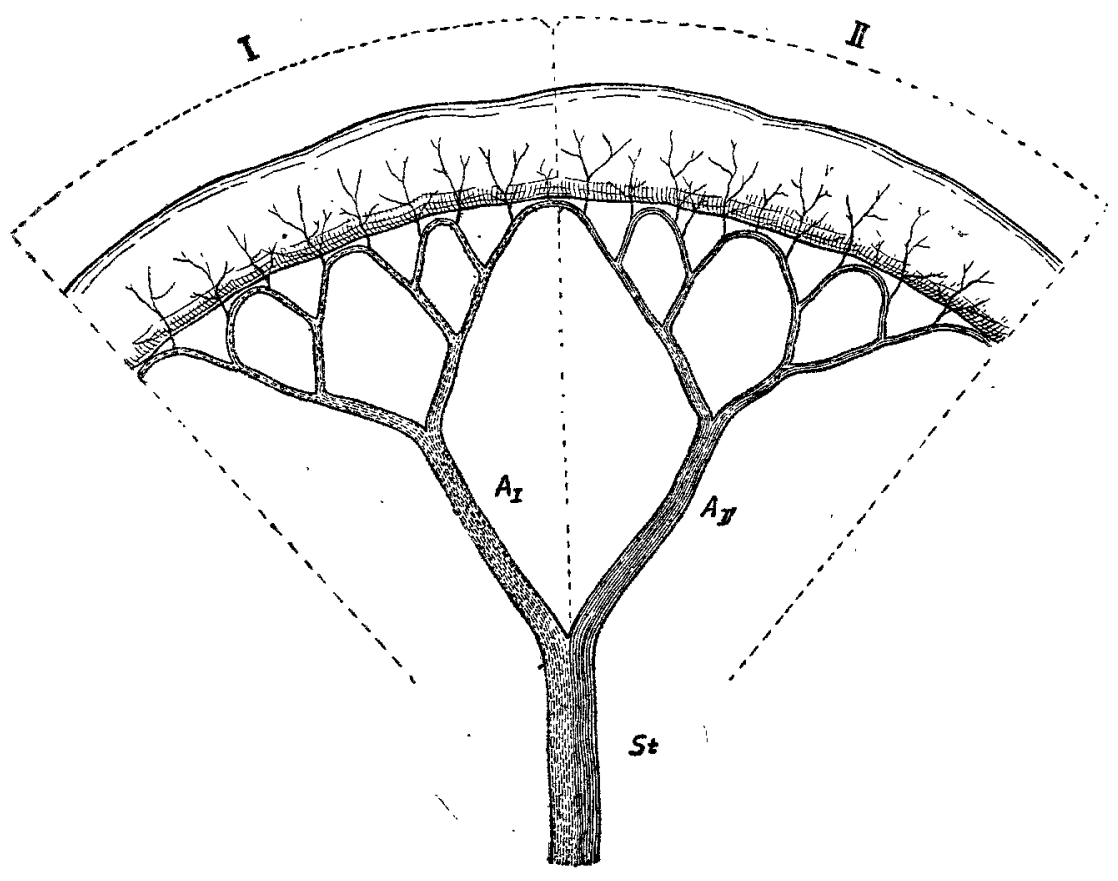

Fig. 1.

(An diesem Schlusse halten wir auch fest, nachdem seit Erscheinen der erwähnten Publikation von Mare $\check{s}^{2}$ ) die Begründung einer

1) W. R. Hess, Die Arterienmuskulatur als „peripheres Herz"? Pflüger's Arch. Bd. 163 S. 555. 1916.

2) Franz Mareš, Der allgemeine Blutstrom und die Förderung der Blutdurchströmung der Organe durch die Tätigkeit ibres Gefässsystemes : I. Förderung des Blutstromes durch aktive Beteiligung der Gefässe am arteriellen Pulse. Pflüger's Arch. Bd. 165 S. 159. 1916. - II. Die Atembewegurgen des Gefässsystemes.' Pfläger's Arch. Bd. 165 S. 194. 1916. - III. Die Grundlagen der herrschenden vasomotorischen Theorien. - IV. Mechanismus des Eigenbetriebes der Blutdurchströmung in verschiedenen Organen. 
gegenteiligen Ansicht versucht worden ist. Die Diskussion źu diesem speziellen Thema soll an anderer Stelle weitergeführt werden.

Die Grundlage aller folgenden Ausführungen ist die Voraussetzung, dass sich die Kreislaufregulierung auf dem Wege der Widerstandsveränderung vollzieht.

Um zu dem gesuchten weiteren Ausbau der Dynamik der Gefässmuskulatur zu gelangen, halten wir uns am besten an die Besprechung eines konkreten Beispielsfallẹs.

Fig. 1 stelle ein in der Kontinuität gedachtes Darmstück dar mit zugehörigem Gefässapparat. Wir denken diesen repräsentiert durch die arteriellen Bahnen, eingedenk der Tatsache, dass es diese sind, welche dursch ihre Muskulatur den ausschlaggebenden Finfluss auf eine regulatorische Umgestaltung der Zirkulationsverhältnisse ausïben.

Das in Fig. 1 dargestellte Darmstück ist zur Betrachtung so ausgeschieden gedacht, dass seine Grenzen mit den Grenzen des Versorgungsgebietes des Stammgefässes $(S t)$ zusammenfallen. Was wir in dieser Abgrenzung nach der Blutversorgung durch ein gemeinschaftliches Muttergefäss vor uns haben, nennen wir weiterhin ein Vers orgungsgebiet oder Strom gebiet.

Entsprechend der zirkulatorischen Zusammengehörigkeit unterscheiden wir an ihm wieder Unterabschnitte, versorgt je durch einen Hauptast $A_{I}$ und $A_{I I}$.

Diese Unter abschnitte nennen wir künftighin Versorgungs bezirke oder Strombezirke.

Den Blutstrom, der sich in das ganze Stromgebiet ergiesst, markieren wir durch Eintragen von "Stromfäden " in den Verlauf der Gefässe (punktierte bzw. ausgezogene feine Linien in den Gefässen). Wir tun dies im Anschluss an die Vorstellung, dass die Vorwärtsbewegung des Blutes in den Gefässen in sogenannter gl leitender Bew egung sart geschieht, wobei die Bewegung aller Flüssigkeitsteilchen parallel der Gefässachse erfolgt. Ein Stromfaden entspricht demnach dem Wege, auf dem ein einzelnes Blutteilchen peripherwärts zieht.

Die Situation, in welcher wir das Darmstück bei Beginn unserer Betrachtung antreffen, sei die der vollkommenen Ruhe, d. h. ohne sekretorische, resorptorische oder motorische Funktion.

Dieser Zustand der Ruhe werde nun dadurch unterbrochen, dass 
von dem oralwärts angeschlossenen Darmabschnitt Speisebrei vorgeschoben wird, welcher vorerst nur in den Darmabschnitt $I$ eintritt. Durch diese Veränderung der Situation ist Anlass gegeben, dass dieser Darmabschnitt beginnt, seine spezifische Funktion auszuüben. Die Funktion ibrerseits ist an eine vermehrte Blutzufuhr gebunden, welche herbeizuführen nun die Aufgabeder Kreislaufregulierung ist.

Nochmals sei hervorgehoben, dass die Steigerung des Blutbedarfes nur das Versorgungsgebiet des Astes $A_{I}$ betrifft, während der Abschnitt $I I$ vorläufig in seinem ursprünglichen Funktionszustand verharrt und deshalb einer Vermehrung der Blutzufuhr nicht bedarf. Dass in Wirklịchkeit der Funktionszustand zweier benachbarter Darmabschnitte nicht derart scharf abgegrenzt ist, wie wir sie zum Zwecke der Analyse annehmen, tut nichts zur Sache. Es soll uns das konkrete Beispiel lediglich eine für die Betätigung: der peripheren Regulierung typische Situation darstellen. Diese Situation charakterisiert sich durch die Notwendigkeit, in einem begrenzten Zirkulationsbezirk die Durchblutung zu verändern.

Zu untersuchen ist jetzt, welche Zustandsäuderung Platz greifen muss, damit der bewirkte Zirkulationseffekt der geforderten Strömungsänderung entspricht.

Zweifellos wirkt im angestrebten Sinne die Erweiterung sämtlicher Gefässe, durch welche hindurch diejenigen Stromfäden ihren Weg nehmen, die sich nach dem Darmabsehnitt $I$ hin aufsplittern. Diese Gefässe sind erstens der Hauptast $A_{I}$ und zweitens alle aus ihm hervorgehenden Zweige. Gleichsinnige Veränderung ihrer Querschnitte bewirkt gleichsinnige Veränderung der Strömung. Z w i s ch en der Muskulatur, welcheentlangdenselben Stromfäden angeordnet ist, besteht also ein synergistisches Verhältnis.

Ob der Wirkungsgrad, mit welcher die Muskulatur die Strömung zil beeinflussen vermag, die gleiche ist, wenn sie in der Wandung des Hauptastes oder der Tochteräste oder deren Zweigen gelegen ist, bleibe vorerst dabingestellt; es ist klar, dass in dieser Beziehung sehr wesentliche Unterschiede bestehen können und unter Umständen auch bestehen. Auf die Untersuchung dieser Frage werden wir später zurückkommen.

Vorher interessiert uns noch die Frage, ob mit der assoziierten 
Betätigung ${ }^{1}$ ) der synergistischen Gefässmuskelgruppe die Möglichkeit. erschöpft ist, dem regulierungsbedürftigen Darmabschnitt $I$ den notwendigen Stromzuwachs zu bringen.

Es ist naheliegend, an die Mitwirkung der Muskulatur des. Stammgefässes $(S t)$ zu denken, weil ja dieselben Stromfäden, welchesich im regulierungshedürftigen Bezirk aufteilen, auch durch das. Stammgefäss hindurch ihren Weg nehmen. Tatsä.chlich ist auch die Zuflussmenge zum Bezirke $I$ dem Einfluss der Muskulatur des Stammgefässes unterworfen und ausserdem noch der Muskulatur aller derjenigen Gefässstrecken, welche sich zwischen Stammgefäss und dem. Blut spendenden Zentrum einschalten. Doch liegen hier insofern besondere Verhältnisse vor, als Stammgefässe und seine zentralwärts gelegenen Vorläufer nicht ausschliesslich Stromfäden für den. regulierungsbedürftigen Bezirk führen, sondern daneben noch solche, welche nach anderen Gebieten abzweigen, in unserem Beispielsfall nach dem Darmabschnitt $I I$. Für die Muskulatur des Stammes $(S t)$ besteht keine Möglichkeit, die Blutversorgung des Abschnittes $I$ und des Abschnittes $I I$ getrennt zu beeinflussen. Der Erfolg ihres aktiven Eingreifen besteht vielmehr in einer Änderung der mittleren. Zuflussmenge für beide Abschnitte zusammen.

Funktionell stelltsich die Muskulatur des Stammes gleichsam zwischen die Muskulatur des zentralen und derjenigen des peripheren Zirkulationsapparates. Es würde viel Interesse bieten, an dieser Stelle die Übergangsbeziehungen zwischen zentralem und peripherem Reguiationsmechanismus näher zu erörtern. Wir müssen aber diese Besprechung in eine spätere Arbeit verweisen, weil sie uns zu weit vom vorgenommenen Thema wegführen würde.

Eine dritte Möglichkeit, dem Darmabschnitt $I$ die notwendige Steigerung der Blutzufuhr zu verschaffen, macht sich geltend, wenn sich die Querschnitte des Astes $A_{I I}$, seiner Töchtergefässe und deren.

1) Unter aktiver 'Betätigung verstehen wir jede aktive Spannungsänderung der Gefässmuskulatur, gleichgültig, ob es sich um Spannungszunahme oder -abnahme handelt. Diese Bezeichnungsweise entspringt der Vorstellung, dass. der physiologische Wert der Gefässmuskelwirkung nicht in der Produktion mechanischer Arbeit liegt, sondern in der Herbeiführung einer Zustandsänderung in den Strömungsbedingungen. Dementsprechend haben wir keinen Grund, entgégengesetzte Zustandsänderungen prinzipiell verschieden zu bewerten. Aktive Betätigung der Gefässmuskulattr ist also jede durch sie bewirkte Querschnittsänderung. , 
Zweigen verengern. Der Einfluss dieser Veränderung vermag sich im Strombezirk $I$ geltend zu machen, obgleich durch diese Gefässe überhaupt keine Stromfäden verlaufen, deren Bestimmungsort der regulierungsbedürftige Bezirk $I$ ist. Die Wirkung ist eine indirekte; sie entfaltet sich dadurch, dass die Art der Aufteilung des vom Stamme $(S t)$ zugeführten Blutstromes vom Verhältnis der Widerstände in den Strombezirken $I$ und $I I$ abhängt. Eine Änderung dieses Verhältnisses kann aber nicht nur durch einen Widerstandswechsel im ersteren, sondern auch im letzteren herbeigeführt werden. Es erscheint die Stromvermehrung zu Darmabschnitt $I$ als Konsequenz der Gefässerweiterung im Strombezirk $I$, ferner als Konsequenz der Gefässverengerung im Strombezirk $I I$. Bei gleichzeitiger Aktion findet die Muskulatur im ersten Bezirk Unterstïtzung durch eine entgegengesetzt gerichtete Aktion der Muskulatur im letzteren Bezirk, gleichsinnige Aktion würde dagegen dem zirkulatorischen Fffekt entgegenarbeiten. Wir finden also zwischen der Muskulatur der beiden Bezirke ein ganz analoges Verhältnis wie zwischen Antagonisten und Synergisten des Skelettmuskelsystems. Nur mit dem Untersehied, dass bei diesem letzteren unmittelbar die Muskelkraft antagonisiert, im Gefässmuskelsystem dagegen der Strömungseffekt, der herbeigeführt wird durch die Querschnittsänderung. Wenn wir hier die in der Dynamik des Skelettmuskelapparates geläufigen Begriffe von Synergismus und Antagonismus auf das Gebiet des Gefässmuskelapparates übertragen, so geschieht es vom Standpunkt dessen Kreislauf regulierenden Fuuktionen. Von diesem Standpunkt gewinnen die genannten Begriffe folgende Bedeutung:

S yn ergismus besteht zwischen denjenigen Teilen der gesamten Gefässmuskulatur, welche dieselb en Stromfäden umspannen in anderen Worten -, welche hinterein ander in das Gefässnetz eingeordnet sind.

Antagonismus besteht zwischen derjenigen Gefässmuskulatur, welche verschiedene Stromfäden umspannt - in anderen Worten -, welche nebeneinander in das Gefässnetz eingeordnet ist.

Eingedenk der Bedeutung der Antagonisten für das Zustandekommen koordinierter und dosierter Bewegungsakte der Skelettmuskelapparatur ist die Frage gegeben, welche Rolle wohl den Antagonisten im Spiele der Gefässmuskeln zukommt. 


\section{b) Die Bedeutung der Antagonistenwirkung für die Ökonomie des Kreislaufes.}

Wir wollen nun versuchen, die zirkulatorischen Konsequenzen einer Assoziation der Antagonisten zur Funktion der Agisten festzustellen. Ein Urteil bierüber wird uns möglich, wenn wir uns in dem auf Seite 447 skizzierten Beispielsfall die Situation jetzt so verändert denken, dass sowohl Abschnitt $I$ als auch Abschnitt $I I$ eine Steigerung dèr Blutzufuhr nötig haben.

Erfolgt die Einstellung auf erhöhten Blutbedarf beider Abschnitte unter Mitwirkung der Antagonisten, so sehen wir sich folgendes Spiel entwickeln:

1. Die Gefässmuskulatur des Strombezirkes $I$ erhält Impulse im Sinne der Quersehnittserweiterung. Dies zum $Z$ weeke der Steigerung der Blutzufuhr nach dem zugehörigen Darmabsehritt $I$. Gleichsinnigen Erfolg erwirken entgegengesetzte, $d . h$. verengernde Impulse zu den Antagonisten, welche im gegebenen Falle die Gefässe des Bezirkes II sind.

2. Die Muskulatur des Bezirkes $I I$ erhält Erweiterungsimpulse, deren $Z$ week es ist, die für diesen Darmabschnitt $I I$ nötige Steigerung der Blutzufuhr zu erwirken. Gleichsinnigen Erfolg soll herbeiführen die gleichzeitige entgegengesetzte Betätigung, der Antagonistengruppe, also im gegebenen Falle Verengerung der Gefässe des Bezirkes $I$.

So sehen wir die Muskulatur eines jeden Bezirkes in zwei getrennten Rollen in Funktion treten, nämlich als $\mathrm{A} g$ is ten zugunsten der Blutversorgung des eigenen Bezirkes, als Antagonisten zugunsten der Versorgung des Nachbarbezirkes. Die beiden Funktionen widerstreiten sich; es kommt zu Rivalität zwischen zwei Interessen. Welehem der beiden die Muskulatur nun wirklich folgt, hängt davon $a b$, mit welcher Intensität die beiden antagonistischen Interessen sich geltend machen. Sie gehorcht dem stärker dosierten Impulse, d. b. zugunsten desjenigen Bezirkes, welcher eine Erhöhung der Blutzufuhr dringender bedarf. Und das Endresultat dieser Rivalität muss derjenige Querschnittszustand sein, bei dem sich die Intensităt der Impulse die Wage hält. Dies ist der Fall, wenn in keinem der beiden rivalisierenden Bezirke das Verlangen nach Steigerung der Blutzufuhr stärker ist als im Nachbargebiet, wenn sich also die Verteilung des vom gemeinschaftlichen Stamme zugeführten Blutstromes im Verhältnis des Blutbedarfes vollzieht.

Inwieweit es dabei zu einer vollk ommenen oder eventuell 
nur partiellen Deckung dès Blutbedarfes beider Abschnitte kommt, hängt davon $a b, o b$ der zur Aufteilung gelangte Blutstrom stark genug ist, beide Abschnitte zu sättigen. Hier nötigenfalls korrigierend einzugreifen, ist Sache der zentralen Regulierung, deren dynamisches Mittel das Herz ist und auch die Gefässmuskulatur, soweit sie die Stromfäden vom Zentrum her bis zu dem Punkte begleitet, wo die Stromfäden nach den beiden rivalisierenden Bezirken auseinandergehen.

Wir wiederholen: Das Eingreifen der (zirkulatorischen) Antagonisten in die Steuerung des Blutstromes gewährleistet einen Verteilungsmodus, welcher den Bluthunger des Gewebes überall a uf den gleichen Grad einstellt. Diese Nivellierung vollzieht sich über den ganzen Organismus, weil sich dieselbe Rivalität, die wir an kleinen Strombezirken besprochen, auch zwischen grossen Gebieten abspielt. schliesslich auch zwischen den Stromgebieten der Hauptstämme, die aus der Aorta hervorgehen.

Und der Sinn einer solchen Nivellierung?

Sie ist der erste Schritt zur Erreichung eines für den Organismus sehr wichtigen Zieles:

Sie teilt nämlich den vom Zentrum gelieferten Blutstrom so auf, dass die ausreichende Ernährung aller Gewebe des ganzen Organismus mit einem kleinst möglichen Gesamtstromvolumen möglich ist.

Dass dem so ist, lässt sich leicht zeigen. Gesetzt den Fall, dass der Bluthunger des Gewebes nicht überall gleichmässig gestillt ist, so sind folgende zwei Fälle möglich: Entweder es ist die vom Zentrum gelieferte Gesamtblutmenge so gross, dass selbst die zirkulatorisch schlechter gestellten Bezirke genügend Blut erhalten, um a us reichend ernährt zu sein. In diesem Falle erhalten naturgemäss die günstiger gestellten Bezirke mehr Blut, als zu ihrer Ernäbrung unbedingt erforderlich. Es kommt in diesen Bezirken zu einem Durchblutungsüberschuss, und der sekundliche Gesamtverbrauch kann nicht das absolute Minimum sein.

Die zweite Möglichkeit ist die, dass die vom Zentrum gelieferte Blutmenge eben ausreicht, den zirkulatorisch begünstigten Bezirken. diejenige Blutmenge zur Verfügung zu stellen, die den Bedarf eben deckt. Dann aber bekommen die relativ schlechter gestellten Bezirke weniger als unbedingt nötig; es kommt zu einer Insuffizienz der Durchblutung, welche nicht bestehen bleiben kann ohne Schaden für das Gewebe. Es muss eine zirkulatorische Korrektur ein- 
treten. Besteht sie darin, dass die Durchblutung ü b e r a 11 gesteigert wird, so haben wir wieder in den anderen Gebieten Durchblutungsüberschuss, was dem Ökonomieprinzip widerspricht. Soll eine Kòrrektur stattfinden ohne jene überflüssige Blutabfuhr nach der Peripherie, so bleibt als einzige. Möglichkeit die lokale Korrektur, welche den Blutstrom nur dort anwachsen lässt, wo das Gewebe an insuffizienter Durchblutung leidet, und welche den Blutstrom so lange anwachsen lässt, bis auch hier dem Blutbedarf eben entsprochen ist, d. h. Gleichstellung mit den übrigen Gewebebezirken erreicht ist. In der Einregulierung auf Gleichstellung in der Absättigung des Bluthungers seben wir also das Mittel, welches überall ausreichendę Durchblutung gewährle istet, ohne dass irgendwo im System ein unökonomischer Durchblutungsüberschuss besteht. Damit ist die Vorbedingung erfüllt für die Einstellung des sekundlichen Gesamtverbrauches an Blut auf das absolute Volumminimum. Dass diese Vorbedingung ausgenützt wird, dafür zu sorgen, ist Sache der Zentralregulierung, auf die wir an dieser Stelle nicht eingehen. Worauf es hier ankommt, ist die Feststellung, dass das Eintreten der Antagonisten in die, motorischen Akte der Kreislaufregulierung diese selbst dem Prinzipe der Ökonomie unterstellt. Auch hierin sei wieder auf eine Analogie zur Dynamik des Skelettmuskelapparates hingewiesen und ferner ein teilweiser Gegensatz der dargelegten Auffassung zu derjenigen festgestellt, welche die Vasokonstriktion in anderen Organen als einen Ausdruck der Druckregulierung ansieht, wie sie zum Beispiel in folgenden Sätzen Tigerstedt's ${ }^{1}$ ) zum Ausdruck kommt.

„Im allgemeinen kann man sagen, dass jeder Körperteil unter normalen Verhältnissen gerade die Blutmenge erhält, welche er nötjg hat, und dass der Körperteil durch Frweiterung seiner Gefässe um so mehr Blut erhält, je kräftiger die in ihm augenblicklich stattfindende Tätigkeit ist. Gleichzeitig werden die Blutgefässe in anderén Körperteilen verengert, und solcherart wird der für das Leben des Körpers notwendige Blutdruck durch ununterbrochene Wechselwirkung $\mathrm{zwischen} \mathrm{den} \mathrm{verschiedenen} \mathrm{Gefässgebieten} \mathrm{unter-}$ halten."

Nicht im Dienste der Blutdruckregulierung erfolgt die reziproke

1) R. Tigerstedt, Lehrbuch der Physiologie des Kreislaufes. Leipzig 1893. 
Innervation in den Konkurrenzoebieten, sondern als Ausdruck eines Mechanismus, welcher die sekundliche Blutabgabe des arteriellen Systems auf ein Minimum beschränkt.

\section{c) Verschiedene mechanische Momente, welche die Kreislauf- regulierung beanspruchen.}

Die Aufgabe der Kreislaufregulierung ist es, dann korrigierend einzugreifen, wenn die Blutversorgung eines bestimmten Gewebebezirkes unter dessen Blutbedarf zurückbleibt.

Als Ursache für das Zustandekommen eines solches Missverhältnisses haben wir bis jetzt ausschliesslich die Zunahme der spezifischen Tätigkeit des Parenchyms und der durch sie bedingten Zunahme des Blutbedarfes ins Auge gefasst.

Es ist von prinzipieller Wichtigkeit für das Verständnis der Kreislaufregulierung, dass ein solches Missverbätnis aber auch noch in anderer Weise entstehen kann, nämlich dadurch, dass bei gleichbleibendem Bedarf die Blutversorgung aus verschiedenen, rein mechanischen Gränden zurückgeht. Gelegenheit für das Eintreten dieser Situation ist reichlich gegeben, und zwar infolge einer unvermeidlichen dynamischen Rückwirkung, welche jeder lokale Regulationsakt auf eine nähere und weitere Umgebung ausübt.

Es lässt sich dies wieder am besten an Hand eines konkreten Beispieles erörtern. Wir können uns an denselben Fall halten, den wir auf S. 447 skizzierten. Er ist dadurch charakterisiert, dass der Strombezirk $I$ sich auf grösseres Stromvolumen einreguliert. Ganz abgesehen von der eventuellen Aktion der Antagonisten macht sich diese Strömungsveränderung auch im Bezirk $I I$ geltend. Es wird ihm durch die Widerstandsvermindelung im Bezirk $I$ ein Teil der normalen Blutzufuhr entzogen. Diese Erschẹnung ist bekannt unter dem Namen der kollateralen Anämie. In ihrem Auftreten haben wir nun den Fall, dass in einem Gewebebezirk die Zirkulation insuffizient werden kann, ohnedass sich in der Tätigkeit des Parenchyms irgend etwas geändert bat. Eine Korrektur ist gleichwohl vonnöten, soll der eingetretene Durehblutungsmangel nicht seine schädlichen Folgen haben.

Die Zahl der Fälle, in denen die Kreislaufregulierung infolge dieser rein mechanisehen Rückwirkung in Anspruch genommen wird, braucht nicht seiten zu sein. Im Gegenteil: wir gehen nicht fehl, 
wenn wir sie als ebenso häufig ansehen wie die Regulierungsakte, die notwendig werden durch eine Veränderung im Funktionszustand des Parenchymes.

Was noch besondere Betonung verdient, ist die Tatsache, dass diese dynamische Rückwirkung auch zwischen Strombezirken besteht, die funktionell voneinander total unabhängig sind. Es weist diese Tatsache darauf hin, dass die Regulierung der Blutversorgung unmöglich von der Innervation der spezifisehen Organtätigkeit ablängig sein kann! Des wolleu wir uns in einem der nachstehenden Abschnitte wieder erinnern.

Und noch eine weitere mechanische Ursache existiert, welche die Forderung nach Selbständigkeit der Kreislaufregulierung unterstützt: Ausser durch Spannungswechsel der Gefässmuskulatur können Quersehnittswechsel an Gefässen auch dadurch herbeigefübrt werden, dass der Innendruck steigt oder fällt, sei es der dynamisch erzeugte Blutdruck oder der hämostatische Druck. Ein Beispiel zum letztgenannten Fall : Bei aufrechter Körperhaltung lastet auf den Gefässen der unteren Extremitäten neben dem dynamisch erzeugten Blutdruck ein beträchtlicher hämostatischer Druck. Unter seiner Einwirkung wird die Gefässwand gedehnt. Wenn wir uns nun plötzlich in horizontale Lage begeben, so wird der hämostatische Druck auf einen viel kleineren Wert herabgesetzt, und es fällt eines der Gefäss dehnenden Momente weg. Die Folge muss sein, dass sich das Gefäss elastisch verengert, was nicht obne Widerstandsänderungen und entsprechender zirkulatorischer Konsequenz geschehen kann. War vorher die Blutversorgung gerade entsprechend dem Blutbedarf eingestellt, so wird sie jetzt insuffizient sein wegen der eingetretenen Verengerung. Für den Widerstand der Gefässbahn besteht kein Unterschied, ob sich die Gefässquerschnitte verengern, weil bei gleichbleibendem Innendruck die Wandspannung durch Muskelwirkung erhöht wird, oder ob sich die Gefässe verengern, weil bei gleichbleibender Wandmuskelspannung der Innendruck plötzlich herabgesetzt wird. Soll die Durchblutung nach der Druckentlastung wieder in die richtigen Grenzen zurückgeführt werden, so muss die elastische Verengerung der Strombahn durch die entsprechende Veränderung der Gefässmuskelspannung kompensiert werden, das heisst die Regulierung in Aktion treten. Auch diese Fälle treten durchaus nicht selten auf. Wir sehen das ein, wenn wir daran denken, wie häufig wir zum Beispiel die Lage unserer 
vorderen Extremitäten ändern. Dies kann nicht geschehen ohne eine tiefgreifende Beeinflussung der hämodynamischen Bedingungen in den zugehörigen Abschnitten des Zirkulationsapparates.

Wir haben somit wieder ein mechanisches Moment kennengelernt, welches die Kreislaufregulierung beansprucht, ohne dass dabei eine Steigerung des Blutbedarfes im Versorgungsgebiete im Spiele wäre.

\section{d) Die Inkonstanz der Gefässmuskelwirkung.}

Im vorhergehenden Abschnitt haben wir gesehen, dass die Forderungen, welchen die Kreislaufregulierung zu genügen hat, viel mannigfaltiger sind, als wir anzunehmen gewohnt waren. Denn sie hat nicht nur den Blutstrom bei Veränderung des Blutbedarfes richtig zu steuern, sondern auch die zirkulatorischen Konsequenzen verschiedener mechanischer Faktoren zu kompensieren.

In diesem Abschnitt werden wir uns davon überzeugen müssen, dass auch die Erfüllung der Forderungen viel komplizierter ist, als man sich bei oberflächlichem Zasehen denkt.

Die Herstellung des Gleichgewichtes zwischen Blutversorgung und Blutbedarf ist nur denkbar, wenn die regulierenden Gefässmuskelaktionen genau dosiert werden können. Mit dieser Dosierung hat es nun aber seine Schwierigkeit, und zwar deshalb, weil ein und derselbe aktive Spannungswechsel der Muskulatur durchaus nicht immer dieselben zirkulatorischen Konsequenzen im Gefolge hat. $\mathrm{Ob}$ die Gefässmuskulatur eine Gefässverengerung entgegen einem hohen oder niedrigen Blutdruck zu erwirken hat, ist durchaus nicht gleichgültig. Je höher der Innendruck, um so grösser muss der aktive Spannungszuwachs sein, wenn eine bestimmte Querschnittsverringerung herbeigeführt werden soll.

Ein weiterer Faktor, welcher die Konstanz im Verbältnis. zwisehen Intensität der Gefässmuskelaktion und der durch sie bewirkten Strömungsänderung stört, ist die von $\mathrm{Fuchs}^{1}$ ) bewiesene Tatsache, dass es für eine bestimmte Durchmesserveränderung eines Gefässes einer verschiedenen Kraft bedarf, je nach dem Grade der Längsspannung, der das Gefäss im Moment unterworfen ist. Da diese Längsspannung speziell bei den Gefässen der Extremitäten

1) Fuchs, l. c. S. 444 . 
je nach den Gelenkstellungen verschieden ist, so wird durch sie ein neuer variabler Faktor in die Querschnittsbestimmung durch die Gefässmuskelwirkung hineingetragen.

Und eine dritte Ursache, welche den zirkulatorischen Erfolg zu einer höchst komplexen Funktion der Gefässmuskelwirkung macht: Sie besteht in der bereits aus anderen Gründen besprochenen mechanischen Rückwirkung der Regulationsakte in Nachbargebieten. Es ist ein wesentlicher Unterschied, ob ein begrenztes Zirkulationsgebiet für sich allein reguliert, oder ob gleichzeitig mit ihm auch Nachbargebiete die Blutversorgung auf ein höheres Niveau einstellen. Es bedarf nämlich einer stärker ausgeprägten Querschnittserweiterung, um eine bestimmte Stromvermehrung zu erwirken, wenn Nachbargebiete gleichzeitig in derselben Regulationsphase stehen als in. der entgegengesetzten.

\section{e) Sensorische Kontrolle der Durchblutung des Gewebes?}

Die dargelegte Analyse der Beziehungen zwischen Gefässmuskelaktion und zirkulatorischem Erfolg bat uns gelehrt, dass ein quantitativ festgelegtes Verhältnis zwischen beiden nicht besteht.

Bei dieser Sachlage ist ein Zweifel sehr angebracht, ob überhaupt ein Regulationsmechanismus physikalisch möglich ist, der all diesen variablen Einflüssen zum Trotz eine genaue Dosierung der Blutzufuhr zustande bringt. So genan, dass im gegebenen Moment ein Gewebe um nichts zu wenig, aber auch um nichts $\mathrm{zu} \cdot$ viel von Blut erhält.

So viel ist gewiss, dass das hierbei zu lösende mechanische Problem ungemein kompliziert ist, wenn nicht, wie gesagt, unlösbar, sofern dem Regulationsmechanismus nicht ein ganz spezielles Hilfsmittel zur Verfügung steht. Ein solches Hilfsmittel wäre ein sensorischer Apparat, der die Durchblutung des Gewebes fortlaufend kontrolliert.

Seine Funktion würde sich nach zwei Richtungen geltend machen:

1. Es würde durch ihn jeder motorische Akt auf seine zirkulatorische Konsequenz nachgeprüt. Dadurch, dass das Resultat der Nachprüfung (auf dem Reflexwege) im weiteren Verlauf der motorischen Aktion zum Ausdruck gelangt, wäre die Grundlage für eine feinste Dosierung gegeben, unbekümmert um dasinkonstante Verhältnis zwischen motorischer Aktion und zirkulatoriscber Konsequenz. 
2. Derselbe sensorische Apparat wäre nicht nur geeignet, die Regulationsakte $\mathrm{zu}$ dosieren, sondern überhaupt zu inszenieren, sobald an irgendeiner Stelle die Durchblutung des Gewebes insuffizient wird.

Diese Möglichkeit vermögen wir. in ihrer Bedeutung richtig einzuschätzen, nachdem wir im vorletzten Abschnitt darauf hingewiesen haben, wie verschiedenartig die Ursachen sind, welche eine regulatorische Umsteuerung dès Blutstromes nötig machen. Ich erinnere an die kollaterale Anämie und an die Quẹschnittsänderung durch Abnahme des Innendruckes. Bei diesen Vorgängen, die ganz unabhängig von der Gewebstätigkeit sind, ist - so scheint es mir - die Einleitung einer Korrektur durch einen dosierten Regulationsakt überhaupt nicht anders denkbar als durch Vermittlung einer spezifischen Sensibilität, deren adäquater Reiz mangelhafte Durchblutung ist. - Es ist dies speziell deshalb hervorzuheben, weil in dieser Beziehung ein Gegensatz besteht zu den Regulationsakten, die durch vermehrte Gewebetätigkeit notwendig werden. Hier wäre wenigstens möglich, dass die Impulse zum Regulationsakt zentralen Ursprunges sind und parallel mit der Innervation des Parenchyms nach der Peripherie gelangen.

Der Gedanke an eine spezifische Sensibilität, welche die Durchblutung des Gewebes kontrolliert, ist durchaus nicht neu. Diese selbe Vorstellung ist es wohl, die den Ausführungen von Roux zugrunde liegt, wenn er von einer Regulation "vom Parenchym aus" spricht.

Ferner kann in diesem Sinne auch das gedeutet werden, was Bayliss in seiner Zusammenfassung unter Punkt $4^{1}$ ) ausführt. Er spricht dort von einer Regulationsmethode, bei welcher eine grössere Blutversorgung durch die Wirkung der Dissimilationsprodukte im bluthungrigen Gewebe herbeigeführt wird. Immerhin stehen wir in einem entschiedenen Gegensatz zu Baylis s', wenn er eine direkte Einnwirkung der Dissimilationsprodukte auf die Gefässe ạnimmt, d. h. als bedingt durch eine Empfindlichkeit der Arteriolen auf $\mathrm{CO}_{2}$. Wir selbst balten die Inszenierung und Dosierung der Blutströmung an die Vermittlung eines spezifischen sensorischen Apparates gebunden.

1) Vgl. S. 441.

Pflüger's Archiv für Physiologie. Bd. 168. 
Auch bei Bier finden wir die Annahme einer Durchblutungssensibilität deutlich ausgesprochen, wenn auch die dafür angewendete Bezeichnủng "Blutgefühl" im Nachtrag zurückgezogen wird; aber auch hier decken sich unsere Auffassungen in bezug auf die Bedeutung derselben nicht. Denn Bier weist dieser Sensibilität nur die Aufgabe zu, bei Unterdrückung einer Blutbahn einen Kollateralkreislauf zu eröffnen, und zwar nur in den Körperabschnitten, in welchen die Blutbahnen häufig Verletzungen und anderen, die Strömung störenden mechanischen inneren Einflüssen ausgesetzt sind ${ }^{2}$ ). Für uns dagegen ist die Durchblutungssensibilität in allen Gebieten des Gefässnetzes eine conditio sine qua non für die restlose Lösung der Aufgaben, die an die Kreislaufregulierung herantreten.

Selbstverständlich sind wir uns darüber klar, dass unsere Durchblutungssensibilität bis jetzt nur ein Postulat darstellt, abgeleitet aus der Dynamik der Gefässmuskulatur.

Es soll nicht Resultate experimenteller Untersuchungen ersetzen, sondern nur zur Direktive werden für Ordnung der in der Literatur bekanntgegebenen Beobachtungen nach neuen Gesichtspunkten, ferner die Direktive für ergänzende eigene Untersuchungen, über welche wir in einer weiteren Arbeit zu diesem Thema berichten werden.

Worauf wir jedoch hier schon kurz hinweisen wollen, ist wiederum die Analogie zur Dynamik der Skelettmuskulatur. Wenn wir die regulatorische Tätigkeit des Gefässmuskelapparates an die Mitwirkung einer spezifischeñ Durchblutungssensibilität gebunden halten, so nehmen wir nichts weiter an, als was für die Funktion des Skelettmuskelapparates bereits als feststehende Tatsache erkannt ist. Auch hier ist bekanntlich eine feinere Dosierung der motorischen Akte ohne ständige sensorische Kontrolle der Bewegungen undenkbar.

Wenn wir den Inhalt der letzten Abschnitte zusammenfassen, so kommen wir zur Vorstellung eines Mechanismus, welcher imstande ist, eine Teilaufgabe der peripheren Kreislaufregulierung restlos zu erfüllen :

Die Regulierung der Blutverteilung auf die verschiedenen Bezirke der zu durehblutenden Gewebe basiert auf der Funktion einer

1) Aug. Bier, Die Entstehung des Kollateralkreislaufes. Arch. f. pathol. Anat. u. Physiol. Bd. 147 S. 256 u. 444 ; ferner Bd. 153 S. 306 u. 434.

2) Vgl. l. c. Bd. 153 S. 458 . 
spezifischen Durchblutungssensibilität. Der adäquate Reiz für dieselbe sind Zustände, die infolge einer mangelhaften Durchblutung auftreten. Ob Anhäufung von Dissimilationsprodukten oder Produkte der Dissimilation bei Sauerstoffmangel bleibe dahingestellt.

Durch die sensorische Erregung wird auf dem Reflexwege ein motorischer Akt ausgelöst, welcher die Korrektur der mangelhaften Durchblutung anstrebt. Die Verknüpfung des korrigierenden motorischen Aktes mit den Leistungen einer sensorischen Kontrolle garantiert die zirkulatorische Korrektur nicht nur für den Fall, dass sie nötig wird infolge Zunahme der Gewebetätigkeit, sondern für jeden Fall. Solche Fälle sind zum Beispiel das Auftreten der kollateralen Anämie und elastischer Querschnittswechsel infolge von Druckveränderungen im System.

Die Leistungen der sensorischen Kontrolle gestatten auch eine genaue Dosierung der Regulationsakte trotz der Inkonstanz des Wirkungsgrades der Gefässmuskulatur. Durch die sensorische Kontrolle wird nämlich der sukzessiv einsetzende Regulationsakt so lange weitergeführt, bis der richtige Durchblutungszustand erreicht ist.

Der motorische Akt selbst besteht, soweit er die Aufgabe der peripheren Kreislaufregulierung erledigt, in einer assoziierten Betätigung zweier Gefässmuskelgruppen:

1. der Agisten. Diese befinden sich in der Wandung derjenigen Gefässe, welche a us s c bliess li c h Stromfäden nach dem regulierungsbedürftigen Bezirke führen;

2. der Antagonisten. Diese befinden sich in den Wandungen der Gefässe, welche kein e Stromfäden nach dem Regulierungsbezirk führen, die also ausschliesslich der Ernährung anderer Gewebebezirke dienen.

Die Bedeutung der Assoziation der Antagonisten liegt in der Erreichung einer möglichst ökonomischen Verteilung des Blutstromes, der von der Zentralregulierung der Peripherie insgesamt für die Aufteilung zur Verfügung gestellt wird.

II.

1. Die dynamischen Beziehungen zwischen der Muskulatur hintereinander gesehalteter Gefässstrecken.

Wir erinnern uns aus Abschnitt I, dass in bezug auf Veränderung des Stromvolumens zwischen der Muskulatur eines Stammgefässes 
und derjenigen der Äste ein sỷnergistisches Verhältnis besteht (gleichsinnige Veränderung des Querschnittes hat gleichsinnigen Strömungseffekt zur Folge). Der Synergismus erstreckt sich, wie auf Seite 451 ausgeführt, über die gesamte Muskulatur, welche entlang den Stromfäden liegt, die sich im regulierungsbedürftigen Bezirk verteilen. Hier wollen wir nun die Frage aufwerfen, ob die Muskulatur der: verschiedenen Teilstrecken in bezug auf ihren Einfluss auf das Strömen unter sich nicht nur qualitativ, sondern auch

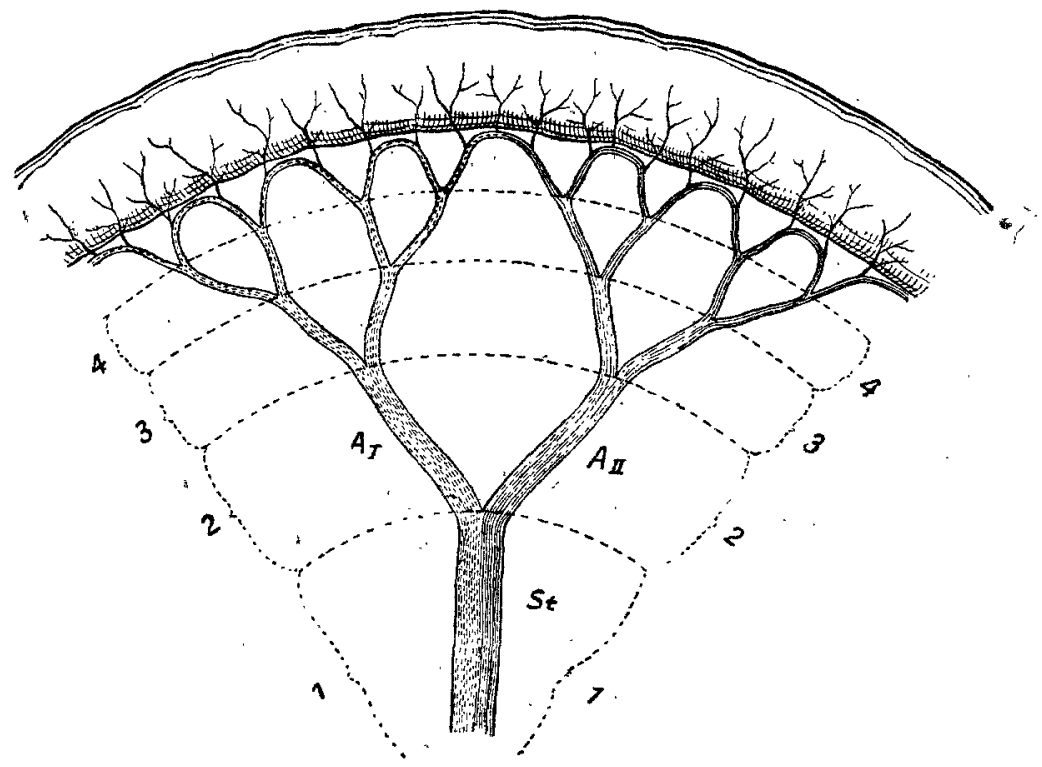

Fig. 2.

quantitativ äquivalent ist oder nicht. Diese Fragestellung lässt sich am konkreten 'Beispiel, wie es in Fig. 2 skizziert ist, näher, erläutern.

Die Skizze stellt das uns bereits von früher her bekannte Darmstück dar, dessen Blutbedarf wegen zunehmender funktioneller Beanspruchung im Anwachsen ist. Um die dadurch notwendig gewordene Vermehrung der Blutzufuhr herbeizuführen, muss (neben der Inszenierung der Antagonistenwirkung) eine Erweiterung der zuführenden Gefässe Platz greifen. Es sind dies die sämtlichen Internodien, welche als direkte und indirekte Zweige aus dem Hauptast $\dot{A}_{I}$ hervorgehen.

Es ist evident, dass der Einfluss der einzelnen Internodien auf 
die Strömung einen sehr verschiedenen Wirkungsgrad haben $\mathrm{kann}$. Es ist zum Beispiel denkbar, dass eine Quersehnittsvermehrung der äussersten Peripherie, das ist in Zone 4 unserer Figur, viel intensivere Wirkung auf die Strömung ausübt als eine genaugleich stark dosierte, das heisst proportionale Querschnittsveränderung der Gefässe zentralwärts, zum Beispiel in Zone 3 oder 2. Anderseits ist es möglich, dass gerade das Gefäss der Zone 2 in seinem Kinfluss auf die Dosierung des Blutstromes überwiegt gegenüber den Internodien der Zonen 3 und 4 . Weitere Möglichkeiten sind eine regulatorische Prävalenz, das heisst ein überwiegender Einfluss der Gefässe irgendeines anderen Abschnittes, der zwischen äusserster Peripherie und dem. Muttergefäss des betreffenden Bezirkes liegt.

Wie in Wirklichkeit die Verhältnisse liegen, darüber sind meines Wissens noch nie Untersuchungen angestellt worden. Meinungsäusserungen, welche in dieses Kapitel fallen, sind allerdings verschiédentlich zu finden. Es scheint, dass die Auffassung verbreitet ist, dass der Haupteinfluss der Gefässmuskulatur auf die Stromdosierung in den präkapillaren Arterien sitzt. Die Vermutung ist deshalb naheliegend, weil man dort den Hauptwiderstand der ganzen arteriellen Bahn vermutet. Die Stelle des Hauptwiderstandes mit der Stelle des grössten Wirkungsgrades der Gefässmuskulatur zu identifizieren, scheint eine logische Folgerung zu sein. Dass dieser Schluss nicht unbedingt richtig zu sein braucht, werden wir später erkennen. Jedenfalls sehen wir jetzt schon ein, dass es notwendig ist, unsere Analyse der Dynamik des Gefässmuskelapparates nun noch in quantitativer Beziehung auszubauen, damit wir in die Lage versetzt sind, die eben aufgeworfene Frage zu beantworten.

\section{Die regulatorische Valenz.}

Die Aufgabe wird leichter, wenn wir uns zuerst an einen schematisch dargestellten einfachsten Fall halten, wie ihn Fig. 3 darbietet.

Wenn in diesem System eine Erhöhung des sekundlichen Stromvolumens bewirkt werden soll, so hat eine Widerstandsänderung Platz zu greifen, und zwar im Sinne einer Herabsetzung. An Stelle des Widerstandes $W$ hat ein neuer Widerstand $W_{1}$ zu treten, wobei $W_{1}$ kleiner als $W$ gedacht ist.

Den Gesamtwiderstand $W$ denken wir uns nun als Summe mehrerer hintereinandergeschalteter Teilwiderstände, wie dies in 


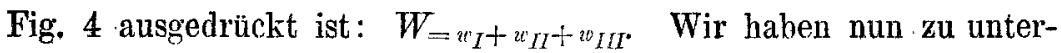
suchen, ob die einzelnen Teilstücke $(I, I I$ und $I I I)$ in bezug auf ihren Einfluss auf die Strömung sich gleichwertig sind oder nicht. Um dies entscheiden zu können, müssen wir die quantitativen Beziehungen kennen, welche zwischen Querschnitt zum Wider-

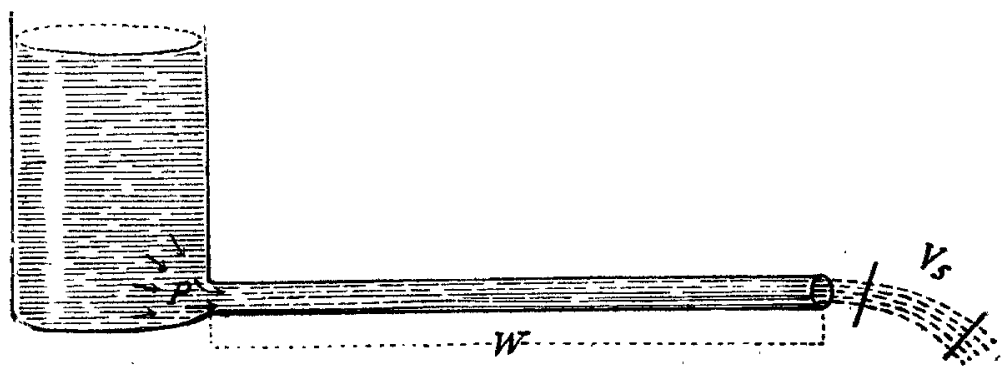

Fig. 3.

stand bestehen. Wir finden diese Beziehungen festgelegt im P o i s e u ill e'schen Strömungsgesetz, dessen Interpretation allerdings nur unter der Voraussetzung zulässig ist, dass die Strömung in Form der sogenannten gleitenden Bewegung vor sich geht. - Wir

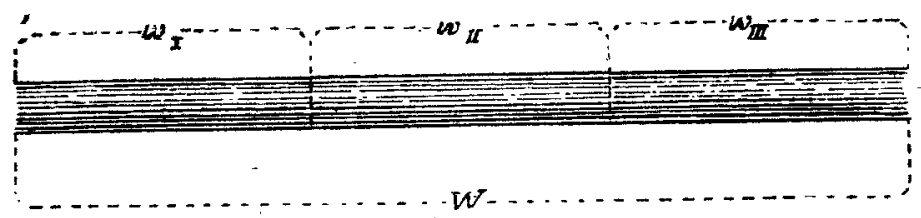

Fig. 4.

werden weiter unten eine Reihe von Gründen und auch direkte experimentelle Beobachtungen kennén lernen, welche uns zeigen, dass diese Voraussetzung im natürlichen Zirkulationssystem mit guter Annäherung zutrifft. Hierauf einzugehen, ist aber erst der Moment gegeben, wenn es sich darum handelt, die Resultate der allgemein gehaltenen physikalischen Orientierung auf die speziellen Verhältnisse des Blutkreislaufes zu übertragen.

Für die Béhandlung der aufgeworfenen Frage sei die Gültigkeit des erwähnten, Gesetzes vorausgesetzt ${ }^{1}$ ). Aus ihm .leiten sich

1) Es schliesst dies die Annahme in sich, dass der Einfluss der Trägheit der bewegten Masse so gering ist, dass er vernachlässigt werden kann. 
wie im Anhang gezeigt, folgende Beziehungen zwischen Widerstand vnd Quersehnitt ab: $\hat{W}=\frac{K}{q^{2}}$

In $K$ sind, wie in der Ableitung der Formel erwähnt, alle diejenigen Faktoren zusammengefasst, welche für die Dynamik der Kreislaufregulierung als invariabel anzusehen sind (Blutviskosität, Länge der Strombahn). Dementsprechend spielt $K$ in der angeführten

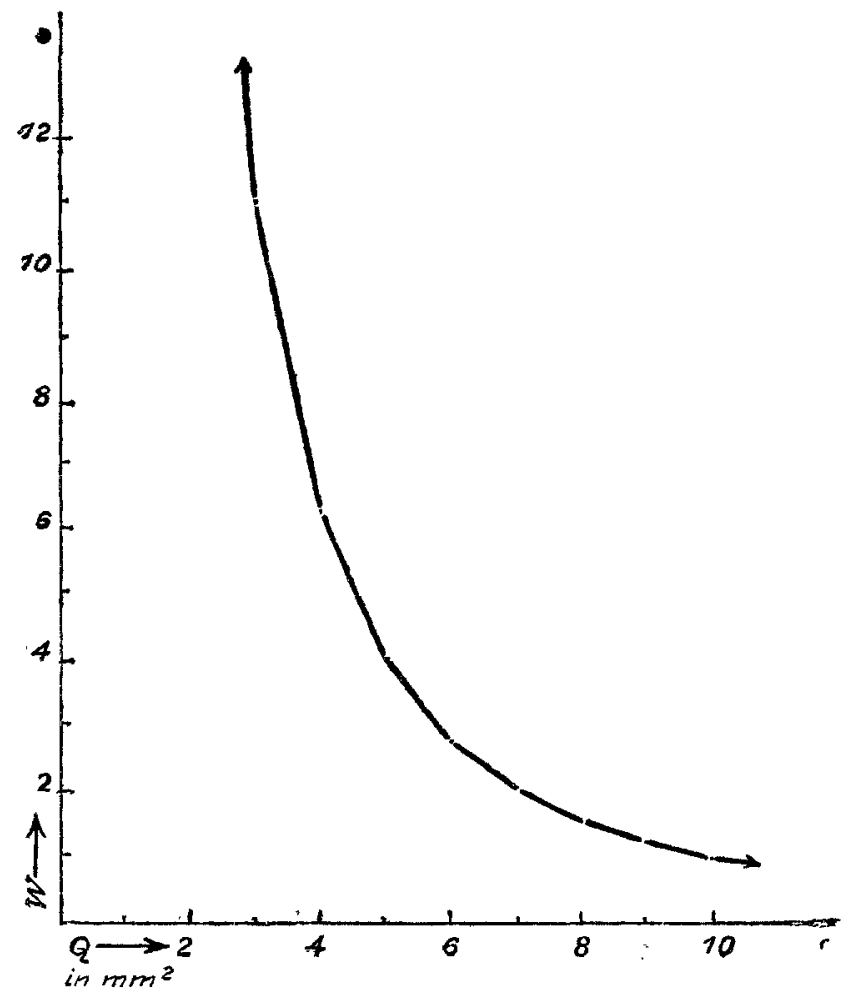

Fig. 5.

Formel die Rolle einer Konstanten. Für den Widerstand besteht somit lediglich Abhängigkeit vom Querschnitt, und zwar ist er umgekehrt proportional zum Quadrat des Querschnittes.

Der Inhalt der gegebenen Formel wird uns äbersichtlicher, wenn wir ibn graphisch darstellen. (Siebe obenstehende Fig. 5.)

Auf der Abszissenachse tragen wir die Querschnitte einer Serie von Röhren auf; die Querschnitte derselben betragen 1 qmm, 2 qmm, 3 qmm ... 10 qmm. Zu jedem dieser Querschnitte gehört ein 
Widerstand, den wir nach der eben abgeleiteten Formel $W=\frac{K}{q^{2}}$ berechnen. Die Maasszahl für die berechneten Widerstände tragen wir in das Koordinatensystem ein als Ordinate zu demjenigen Abszissenwert, zu dem der betreffende. Widerstand berechnet ist. Wir gewinnen dadurch ein Bild, wie es Fig. 5 darstellt. Der Widerstand, welcher einem Querschnitt von $1 \mathrm{qmm}$ entspricht, ist dabei als relative Masseinheit $=100$. angenommen.

Aus der so erhaltenen Kurve gehen zwei Tatsachen hervor:

1. Die Ordinaten werden mit wachsender Abszisse kleiner, d. h. der Widerstand nimmt mit wachsendem Querschnitt ab Es ist dies die bekannte Tatsache, über die wir weiter kein Wort zu verlieren baben.

2. Die Ordinaten zu zwei benachbarten Abszissenpunkten unterscheiden sich durch einen um so grösseren Betrag, je geringer der absolute Wert der verglichenen Querschnitte ist. Dies bedentet, dass der Einfluss der Querschnittsgestaltung auf den Widerstand um so stärker ausgesprochen ist, je enger die Strombahn ist.

Es geht dies besonders deutlich aus der Kurve Fig. 6 hervor.

Es ist dasselbe Kurvenbild wie Fig. 5 . Ihr eingefügt sind abernoch die mit $D_{3} D_{5} D_{7}$ bezeichneten Strecken. Die Länge dieser Strecken entspricht der Differenz der Ordinaten, welche zu zwei aufeinanderfolgenden Abszissenwerten gehören. Sie stellen somit eine Maassstrecke für die Widerstandsändernng dar, die mit der Querschnittsänderung um eine Einheit verbunden ist.

$D_{3}$ ist zum Beispiel die relative Maassstrecke, d. h. proportional dem Widerstandsunterschied zwischen einem Rohr von $3 \mathrm{qmm}$ und einem Rohr von $4 \mathrm{qmm}$ Querschnitt. $D_{7}$ ist die Massstrecke für die Widerstandsänderung, welche statt hat, wenn wir vom Querschnitt 7 qmm zu Querschnitt 8 qmm übergehen. .

Aus der graphischen Darstellung ist ersichtlich; wie sehr verschieden diese Widerstandsänderungen ausfallen, obgleich sie sich immer auf denselben absoluten Betrag der Querschnittsänderung beziehen, nämlich auf eine Änderung um eine Querschnittseinheit. Gros s sind die Widerstandsunterschiede $\mathrm{zwischen}$ zwei um eine Quersehnittseinheit differierenden engen Röbren; klein sind die Unterschiede 
zwischen zwei um die gleiche Einheit differierenden weiten Röhren.

Wo essich also darum handelt, durch Querschnittsänderung eine Widerstandsänderung herbeizuführen, wird dies in erster Linie dureh Querschnittswechsel der engsten Stellen geschehen können.

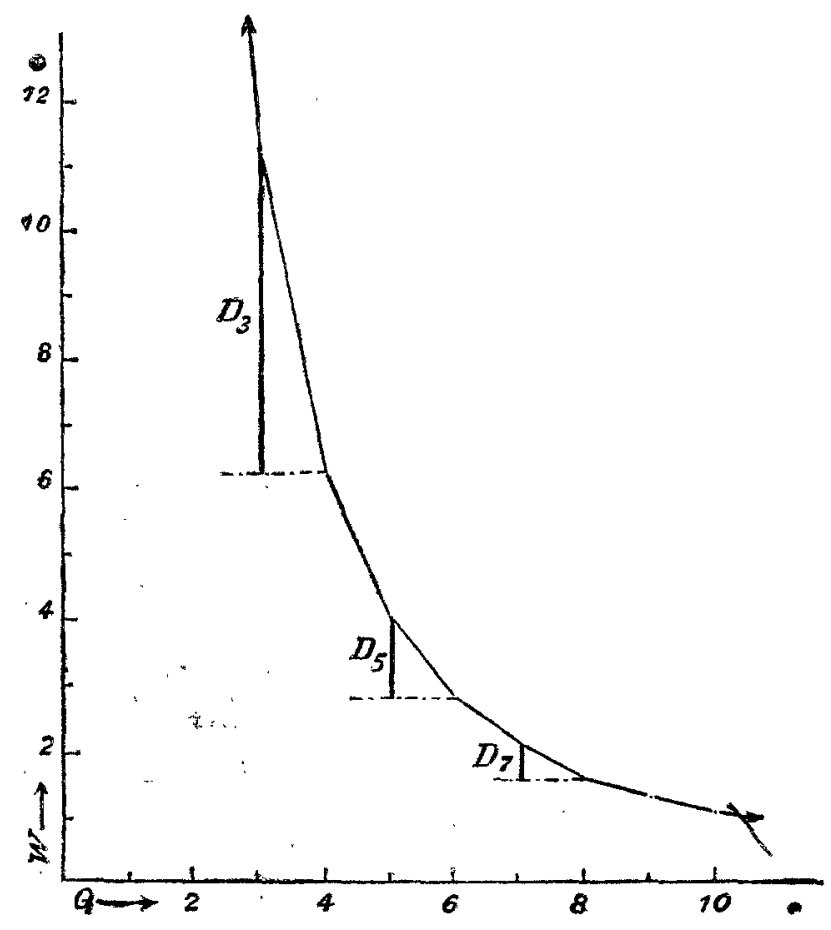

Fig. 6.

Enge Gefässe besitzen, wie wir uns ausdrücken wollen, eine grössere regulatorische Wertigkeit, die grössere regulatorische Valenz. - Um kein Missverständnis aufkommen zu lassen, sei gleich hinzugefügt, dass der Satz in dieser Allgemeinheit nicht auf das Gefässsystem anwéndbar ist. Denn er ist abgeleitet auf Grund der Voraussetzung, dass durch die engen Querschnitte das gleiche Sekundenvolumen getrieben wird wie durch die weiten. Diese Voraussetzung trifft nun für ein verzweigtes Röhrensystem nicht zu. Der Hinweis ist doppelt gerechtfertigt wegen der bereits erwähnten 
Auffassung, dass die Arteriolen wegen ihrer geringen Querschnitte einen ausschlaggebenden Einfluss auf die Widerstandsänderungen des Gefässnetzes besitzen müssten. - Ein Vergleich zwischen Abschnitten der Strombahn, die unter Aufteilung des Stromvolumens auseinander hervorgehen, setzt voraus, dass wir diesen Faktor in unsere Analyse einbeziehen. Bevor. wir hierzu übergehen, wollen wir zur deutlicheren Umschreibung den Begriff, den wir unter der Bezeichnung "regulatorische Valenz" in die Dynamik der Kreislaufregulierung einführeu, an nachstehenden Skizzen (Fig. 7-11) noch genauer erläutern.

Fig. 7 veranschaulicht eine unverzweigte Strombahn, welche aus drei verschiedenen Teilstücken zusammengesetzt ist, deren jedes sich von den übrigen unabhängig im Querschnitt verändern lasse. Weil die Strombahn unverzweigt ist, passiert natürlich durch jeden Querschnitt das gleiche Sekundenvolumen. Es lässt sich somit der auf Seite 467 ausgesprochene Satz auf die hier dargebotenen Verhältnisse anwenden, d. h. es hat eine bestimmte $Q u e r s c h n i t t s-$ änderung einen um so ausgesprocheneren Einfluss auf den Widerstand der Strombahn, je kleiner der Querschnitt ist, an dem die Querschnittsänderung vollzogen wird.

In dem durch Fig. 7 skizzierten Fall zeichnet sich das Teilstück $I I$ vor seinen Nachbarabschnitten dadurch aus, dass es enger ist. Wenn es gilt; den Gesamtwiderstand der ganzen Strombahn herabzusetzen, so ist nach dem Gesagten hierzu in erster Linie eine Querschnittserweiterung geeignet, die am Teilstück $I I$ ansetzt; dieses Stück besitzt dị grösste regulatorische Valenz. Ein bestimmter Querschnittszu wachs erwirkt hier in bezug auf Widerstandsverminderung den grösseren Effekt" als an irgend einer anderen Stelle der Strombahn.

Setzt nun eine regulatorische Querschnittsvermehrung an Abschnitt $I I$ tatsächlich an, dann büsst er freilich mit dem Zustandekommen der Erweiterung seine regulatorische Prävalenz ein. Sie ist vollends verloren gegangen in dem Moment, in welchem der Abschnitt II gleich weit wie die übrigen Abschnitte geworden, weil mit dem Ausgleich des Quersehnites auch eine Nivellierung, das heisst eine Gleichstellung in bezug auf die regulatorische Valenz erfolgt ist. 
Ein anderes Beispiel:

Wir haben in Fig. 8 eine Strombahn vor uns, in welcher sich der A bschnitt $I I$ vor den übrigen dadurch auszeichnet, dass er weiter ist als seine Nachbarabschnitte. Wenn von der so konfigurierten Strombahn eine Erhöhung der Sekundenleistung verlangt wird, so sehen wir jetzt den Erfolg einer Querschnittszunahme des Teilstückes $I I$ hinter dem Strömungseffekt einer gleieh grossen Querschnittsänderung

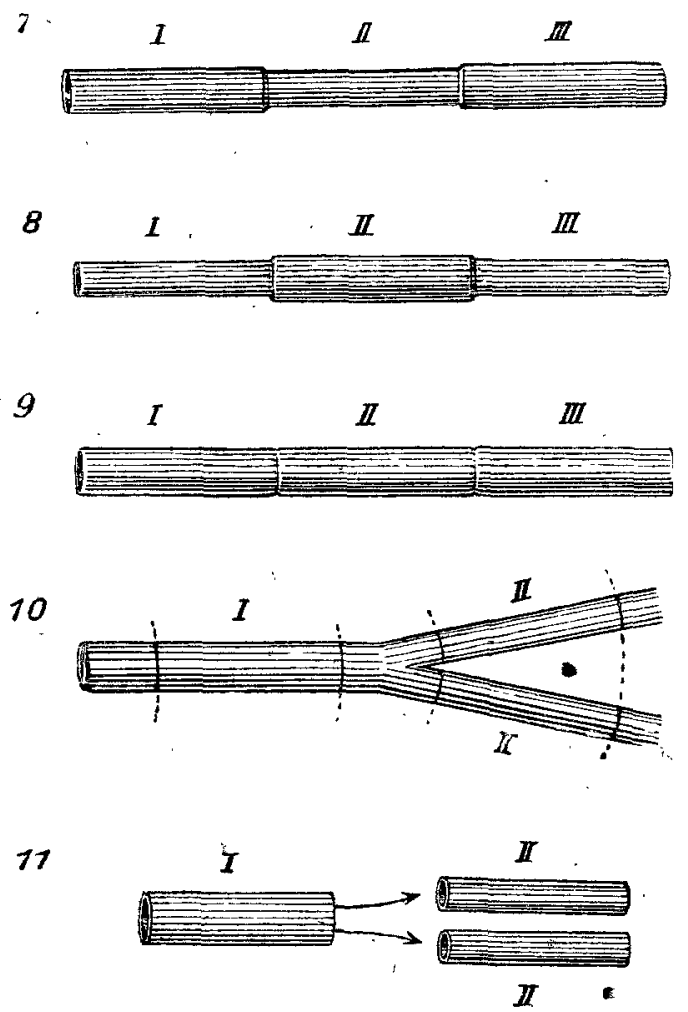

Fig. $7-11$.

seiner Nachbarn zurückstehen. Der gleiche Zuwachs bei $I I$ angebracht, setzt den Gesamtwiderstand weniger herab, als wenn der Zuwachs dem Teilstuck $I$ oder III zugeteilt wird; denn engere Stellen besitzen die grössere regulatorische Valenz.

Lassen wir nun die regulatorische Erweiterung wirklich in dem Abschnitt $I$ und $I I I$ Platz greifen, so büssen sie mit der Erweiterung ihre regulatorische Prävalenz ein; sobald sie dem Querschnitt von Abschnitt $I I$ erreicht haben, ist wieder mit dem Querschnittsausgleich Nivellierung der regulatorischen Valenz erfolgt. 
Und nun noch ein drittes Beispiel:

In Figur 9 haben wir eine zusammengesetzte Strombahn vor uns, in welcher alle Teilstücke g] eichen Querschnitt besitzen. Die Gleichheit kann von vornherein bestanden haben, oder sie entstand unter dem Einflúss einer Regulierung, wie sie an Beispiel 1 und 2 erläutert ist (Fig. 7 und 8).

Wo wird hier ein neuer Regulationsakt am besten angreifen? Bei dieser Strombahn ist, weil Querschnittsgleichheit aller Abschnitte besteht, die regulatorische Valenz überall gleich. Wenn eine Vermebrung des Sekundenvolumens gefordert wird, so ist es auch, quantitativ betrachtet, ohne Belang, an welchem der Abschnifte sich die regulierende Erweiterung vollzieht. Aber von dem Augenblick an, wo er sich an einem Abschnitt vollzogen hat, tritt das betreffende Teilstück in bezug auf regulatorische Valenz gegenüber den Nachbarabsehnitten zurück, weil es jetzt diese an, Querschnitt übertrifft. Die Fortsetzung der Regulation findet einen günstigeren Angriffspunkt in den zurückgebliebenen Teilen. Erst wenn auch diese um einen gleichen Betrag erweitert worden sind, besteht wieder regulatorische Gleichwertigkeit, und erst von jetzt ab kann auch der erst erweiterte Abschnitt wieder Ort einer Regulation sein.

Ziehen wir nuñ das Fazit aus den angeführten Beispielen, so sehen wir eine Tatsache hervortreten:

Wenn der Widerstand einer Strombahn herabgesetzt werden soll, und wenn für die Durchführung der hierzu nötigen Querschnittserhöhung diejenigen Stellen der ganzen Strombahn gewählt werden, die den grössten Einfluss auf den Gesamtwiderstand besitzen, so resultiert in jedem Falle eine Nivellierung aller Querschnitte auf die gleiche regulatorische Valenz. Für die ungeteilte Strombahn ist diese Nivellierung erreicht, wenn überall Querschnittsgleichheit besteht.

Dies ist nicht das einzige Resultat, zu welchem wir geführt werden.

Für unser spezielles Thema nicht weniger wichtig sind folgende zwei Tatsachen:

1. Wennzum $Z$ wecke der Widerstandsverminderung die hierfür nötige Querschnittserweiterung an derjenigen Stelle ansetzt, wo der Einfluss der Querschnittsgestaltung auf den Widerstand die grösste 
Wirkung hat, so wird ein bestimmter Betrag einer Widerstandsänderung mit dem kleinstmöglichen Querschnittsuwachs erreicht.

2. Das Endresultat dieser Art Widerstandsregulierung ist diejenige Konfiguration der Strombahn, welche für einengegebenen mittleren Querschnitt den k]einstmöglichen Widerstand darbietet.

Der erste dieser beiden Sätze ist die logische Folgerung aus der gemachten Annahme; denn wenn eine Querschnittserweiterung so vollzogen wird, dass auf eine bestimmte Querschnittszunahme die grösstmögliche Widerstandsverminderung einsetzt, so bedarf es anderseits des kleinstmöglichen Querschnittszuwachses, um eine bestimmte Widerstandsverminderung zu bewirken.

Die Richtigkeit des zweiten Satzes erkennen wir, wenn wir uns die Strombahn entstanden denken aus einer Strombahnanlage mit feinsten Querschnitten. Die Form, der wir im Moment der Prüfung gegenüberstehen, lassen wir dadurch zustande kommen, dass wir die Querschnitte der einzelnen Teilstücke sukzessive wachsen lassen. Dabei lokalisieren wir das Wachstum in jedem Moment an die Stelle, welche momentan die grösste regulatorische Valenz aufweist. Jeder Zuwachs un eine Querschnittseinheit hat dementsprechend den grösstmöglichen widerstandsvermindernden Effekt im Gefolge.

Dieses muss auch gelten für die Summe aller sich folgenden Querschnittszunahmen, d. h. mit dem Gesamtquerschnittszuwachs hat das System die grösstmögliche Verminderung des Gesamtwiderstandes erfahren. Jede andere Querschmittsverteilung als die auf die Nivellierung der regulatorischen Valenz gerichtete ist mit einem "weniger stark herabgesetzten", das heisst einem grösseren Widerstand behaftet! Im übrigen sei auf die Beweisführung im Anhang verwiesen (Ziffer 4).

Inwiefern nun diese angeführten physikalischen Regeln für die Regulierung des Blutkreisla ufes Bedeutung haben, davon werden wir noch eingehend sprechen. - Soviel wird uns schon jetzt deutlich, dass ein mit der kleinsten Querschnittsänderung reguliertes Strömungssystem der Forderung der Energieökonomie am besten entspricht!

Bevor wir weiter auf diesen Punkt eingehen, haben wir unsere physikalische Analyse noch um einen Schritt weiterzufübren und die Vèrhältnisse zu untersuchen, welche eine verzweigte Strombah n darbieten. 
Die zu untersuchenden Verhältnisse werden dabei komplizierter; wir können aber einem Eingehen auf sie nicht ausweichen, wenn wir uns der vorgenommenen Aufgabe entledigen wollen und die physikalische Grundlage für die experimentelle Behandlung der Kreislaufregulierung gewinnen wollen.

Am einfachsten liegen die Verhältnisse bei symmetrischer Zweiteilung der Strombahn, wo also die aus der Aufteilung des Stammes hervorgehenden Äste gleich sind; Fig. 10 skizziert den Fall (siehe Fig. 10 auf S. 469).

Das „wesentliche" Merkmal dieses Systems ist der Umstand, dass die Flüssigkeit in ihrem ersten Verlauf in einem einheitlichen Strombett geführt wird (dem Stamm); in einem zweiten Abschnitt des Systems wird das Strombett durch zwei getrennte Rohre dargestellt, von denen beide zusammen dieselbe Flüssigkeitsmenge wie der Stamm führen. Die verschiedene Verlaufsrichtung, der getrennten Bahnen tut hier nichts zur Sache, wo es sich um die Untersuchung der Beziehung zwischen Widerstands- und Querschnittsänderung handelt.

Diese Art von Strombahn ist in Fig. 11 S. 469 schematisiert. Den Teil mit einheitlicher Strombahn bezeichnen wir mit Abschnitt $I$ (Stamm), den Teil mit getrennter Strombahn mit Abschnitt $I I$ (Äste).

Wenn an die zirkulatorische Leistung dieses sich verzweigenden „Systems" erhöhte Anforderungen gestellt werden, so frägt es sich nun wieder, $o b$ zwischen den beiden Abschnitten Gleichwertigkeit besteht in bezug auf ibren Einfluss auf die Strömung, oder ob den beiden Abschnitten eine versehiedene regulatorische Valenz zukommt.

Man möchte vermuten, dass es die Äste sind, weìche den grösseren Einfluss auf den Widerstand des Systems besitzen, weil sie die kleineren Rohrquerschnitte aufweisen.

Der Schluss in dieser Form ist falsch, weil wir dabei den Umstand nicht berücksichtigen, dass mit der Teilung der Strombahn auch eine Teilung des Stromvolumens einhergeht.

Was dies für Folgen hat, müssen wir erst feststellen. Diese Feststellung ist eine rein rechnerische. Sache, welche in den Anhang (S. 488 Ziff. 3) verwiesen ist. Die Rechnung führt zu dem Resultat, dass zwischen Stamm und Ästen dann Übereinstimmung in bezug auf regulatorische Valenzbesteht, wenn die Summe der Astquersehnitte das 1,26fache des St a m mquerschnittes $(1,26=\sqrt[3]{2})$ beträgt. 
Ist ihre Querschnittssumme kleiner, so haben die Äste die grösser e regulatorische Valenz. Eine regulatorische Widerstandsverminderung hätte alṣo an den Ästen anżugreifen, wenn die Regulation mit dem kleinsten Querschnițtszuwachs vollzogen werden sọll. Dies gilt so lange, als die regulatorische Erweiterung bis zum genanzten Verbältnis herangeführt hat.

Ist die Summe der Astquerschnitte grösser als das 1,26 fache des Stammquerschnittes, so prävaliert der Stamm im Einfluss auf die Widerstandsgestaltung. Aber auch hier vermindert natürlich das Einsetzen der regulierenden Erweiterung die Prầvalenz, und auch hier ist sie vollends verschwunden im Moment, wo wieder das Verhältnis $1: 1,26$ erreicht ist, weil eben bei diesem Verhältnis Gleichwertigkeit besteht. Die Fortsetzung regulatorischer Widerstandsänderung muss, wenn sie auch fernerhin mit der kleinsten Querschnittsänderung erfolgen soll, gleichzeitig an Stamm und Ästen angreifen. Und weiterhin ist es Erfordernis, dass der Querschnittszuwachs, den die beiden Äste zusammen erfahren, zum Querschnittsuwachs des Stammes selbst nach dem Verhältnis 1,26:1 dosiert sind, weil nur so die Übereinstimmung der regulatorischen Valenz von Stamm und Ästen bestehen bleiben kann.

Es sei betont, dass die aufgeführte Zahl 1,26 nur für einen symmetrischen Verzweigungsmodus Geltung hat.

Für Aufteilung eines Stammes in zwei ungleiche Äste würde sich ein anderer Verhältniswert berechnen. Auf die absolute Grösse dieses Quotienten soll es jetzt aber überhaupt nicht ankommen. Worauf wir Gewicht legen, ist die Konstatierung, dass einem ganz bestimmten Querschnittsverhältnis zwischen Stamm und Ästen zugesteuert wird, wenn in der Strömungsregulierung die Tondenz herrscht, eine Widerstandsänderung durch die kleinstmögliche Querschnittsänderung herbeizuführen. Dies Verhältnis ist erreicht, wenn Stamm und Äste und auch die Zweige der Äste den gleichen Wirkungsgrad auf die Widerstandsgestaltung haben, d. h. wenn die regulatorische Valenz bei sämtlichen Teilstrecken entlang der Strombahn auf denselben Wert nivelliert ist.

Die mathematische Präzisierung eines Systems, das diese Bedingung erfüllt, sind identische Differentialquotienten von Widerstand nach Querschnitt an allen sich folgenden Querschnitten der Strom- 
bahn. Gemäss den auf S. 471 gegebenen Ausführungen haben wir hierin gleichzeitig das Merkmal für ein Strömungssystem, welches bei gegebenem mittleren Querschnitt den kleinstmöglichen Widerstand aufweist.

Diese Beziehungen sind von fundamentaler Bedeutung für Bau und Funktion des Blutgefässapparates, d. h. sofern sich die Resultate unserer mechanischen Analyse auf das lebende Blutgefässsystem anwenden lassen.

Es bleibt also die Frage zu erörtern, ob eine Übertragung der Resultate zulässig ist im Rahmen der Fehlergrenze, die für biologische Gesetze beansprucht werden kann. Entseheidend hierfür ist der Umstand, ob wir bei unseren Ableitungen Voraussetzungen gemacht, die für das lebende Gefässsystem stimmen oder nicht!

\section{Die Prüfung unserer Voraussetzungen auf ihre Gültigkeit im Blutgefässsystem.}

Ausgegangen sind wir von folgenden Voraussetzungen:

a) Der Gesamtwiderstand einer aus verschiedenen hintereinandergeschaltetèn Abschnitten zusammengesetzten Strombahn ist gleich der Summe einzelner Teilwiderstände: $W=w_{I}+w_{I I}+w_{I I I} \ldots$.

b) Der Gesamtwiderstand einer aus parallelgeschalteten Abschnitten zusammengesetzten Strombahn berechnet sich nach der Formel : $\frac{1}{W}=\frac{1}{w_{I}}+\frac{1}{w_{I I}}$.

c) Der.Widerstand eines röhrenförmigen Gebildes steht im umgekehrt proportionalen Verhältnis zum Quadrat des Querschnittes (Teilinhalt der Po is euille'schen Formel): $W=\frac{K}{q^{2}}$.

Über die Anwendbarkeit der Voraussetzung a) und b) auf den Blutkreislauf kann kein Zweifel sein, da es sich um das bekannte Widerstandsgesetz handelt.

Nicht von vornherein sicher. ist dagegen die Übertragbarkeit der Voraussetzung. e). Sie hängt davon $a b$, ob das Strömen des Blutes im gesunden Gefässsystem nach der Poi s e uille'schen Regel erfolgt oder nicht. Die Ansichten hierüber gehen auseinander ${ }^{1}$ ).

1) Vgl. Nagel, Handb. d. Physiol. Bd. 1 S. 764; ferner Tigerstedt, Der arterielle Blutdruck. Ergebn. d. Physigl. Bd. 6 S. 299. 
Ich selbst habe mich schon bei früherer Gelegenheit ${ }^{1}$ ) zu diesem Thema geäussert, die Ansicht vertretend, dass eine Entscheidung dieser Frage für die theoretische Hämodynamik dringend zu wünschen ist. Ich verweise auf die zitierten Ausführungen. Hier soll es genügen, wenn ich die hauptsächlichsten Punkte wiederhole:

1. Das Poiseuille'sche Gesetz ist durch Theorie und Experiment innerhalb sehr weiter Grenzen als für das Strömen von Flüssigkeit maassgebend festgestellt. Es ist eine direkte Konsequenz des Strömens in Form geradliniger Fortbewegung der Flüssigkeitsteilchen, der sogenannten gleitenden Bewegung.

"Gleitende" Bewegung ist gegenüber der sogenannten „turbulenten". diejenige Strömungsform, welche die kleinere Reibung bedingt; vom Standpunkt dèr Energieökonomie ist sie günstiger.

Die Grenze, ausserhalb welcher das Gesetz versagt, ist wiederum gesetzmässig fixiert, und zwar durch die Formel Reynold' $\mathrm{s}^{2}$ ). Der Grund für das Versagen des Gesetzes ist das Auftreten von Wirbeln. welche die Flüssigkeitsteilchen von der geradlinigen Bewegung ablenken. Die Rey nold'sche Formel ist experimentel sichergestellt. Ihre Anwendung auf das Strömen des Blutes lehrt ${ }^{3}$ ), dass es unter normalen Verhältnissen höchstens in den weiten Gefässen zu turbulenter Strömungsart kommen kann (vgl. Reynold'sche Formel im Anhang). Die Umstände, die in der Aorta und deren weitesten Ästen turbulente Strömung herbeiführen, sind niedrige Blutviskosität und beschleunigte Zirkulation. Irrtumlich ist die Beschränkung der Po is euille'schen Formel auf Röhren von weniger als $3 \mathrm{~mm}$ Durchmesser. Die Untersuchungen Poiseuille's, auf die Tigerstedt ${ }^{4}$ ) bei seinen diesbezüglichen Angaben verweist, sind durch die Untersuchungen Reynold's überholt und korrigiert.

1) W. R. Hess, Gehorcht das Blut dem allgemeinen Strömungsgesetz von Flüssigkeiten? Pflüger's Arch. Bd. 162 S. 187. 1915; ferner W. R. Hess, Reibungswiderstand des Blutes und Poiseuille'sches Gesetz. Zeitschr. f. klin. Med. Bd. 71 H. 5 u. 6, Bd. 74 H. 5 u. 6; ferner W. R. Hess, Der Strömungswiderstand des Blutes gegenüber kleinen Druckwerten. Arch. f. (Anat. u.) Physiol. 1912 S. 197.

2) Vgl. Kohlrausch, Lehrb. d. prakt. Physik, 12. Aufl., S. 266. 1914.

3) W. R. Hess, Viskosität des Blutes und Herzarbeit. Vierteljahrschr. d. Naturforsch. Gesellsch. Zürich 1916.

4) Tigerstedt, Der arterielle Blatdruck. Ergebn. d. Physiol. Bd, 6 S. 299. Pflüger's Arehiv für Physiologie. Bd. 16s. 
3. Der Suspensionscharakter des Blutes ist nịcht imstande, nennenswerte Abweichungen zu "verursachen. In feinsten Gefässeì freilich ist eine Störung des Gesetzes durch die korpuskulären. Elemente zu erwarten. $\mathrm{Zu}$ beachten ist aber, dass das Experiment. an Glaskapillaren zeigt, dass Lichtweiten von $0,1 \mathrm{~mm} \mathrm{noch}$ in Bereich des Gesetzes fallen (bei Blut als strömende Flüssigkeit). Wegen der Störungen des $\mathrm{P}_{0}$ i s eu ille'schen Gesetzes. durch hiohäsionserseheinung verweise ich auf meine diesbezüglichen Untersuchungen ${ }^{1}$ ).

4. Verzweigungen und diskontinuierliches Strömen begünstigen das Zustandekommen von' Wirbeln in weiten Gefässen und damit Abweichungen vom Poisseuille'schen Gesetz. Die Wirbelbildungfindet aber anderseits in der hohen Viskosität eine sehr wirksame Dämpfung, welcher Faktor überbaupt für die Aufrechterhaltung der gleitenden Strömungsform des zirkulierenden Blutes. einen durchschlagenden Einfluss hat (vgl. Reynold'sche Formel im Anhang, Ziffer 6).

Den aufgeführten theoretisehen Gründen lassen sich nun noch direkte Beobachtungen an die Seite stellen. Wir kennen die Erscheinung, dass ein von Wirbeln durchsetzter Flüssigkeitsstrom ein Geräusch wahrnehmen lässt. Sehr bemerkenswert ist bei dieser Sachlage die Tatsache, dass die Auskultation von Gefässen, zum Beispiel Arteria cruralis, am Mensch oder Hund keine Spur von. Geräusch verrät. Ja selbst bei Auskultation einer freigelegten Aorta descendens thoracica eines ausgewachsenen Bernhardinerhundes. ist, wie ich $\mathrm{zu}$ konstatieren Gelegenheit hatte, keine Spur von Strömungsgeräusch hörbar! Wenn Geräusche an Gefässen auftreten, so liegen immer besondere Umstände vor, zum Beispiel wenn wir das Gefäss durch Druck so weit deformieren, dass eine wesentliche Verengerung des Lumens eintritt und dadurch eine "Stromschnelle". Oder wenn wir infolge künstlicher Erweiterung der peripheren Gefässe eine ungewöhnlich hohe Strömungsgeschwindigkeit im zuführenden Stammgefäss haben (Bier). Auch ein Unterbruch des gleichmässigen Querśchnittes eines Gefässes durch abnorme Erweiterung (Aneurysma) lässt Wirbel entstehen.

In jedem Falle kündet sich das Auftreten von Wirbeln durch Strömungsgeräusche an. Damit ist der Schluss gegeben, dass d as

1) Arch. f. (Anat. u.) Physiol. 1912 S. 197. 
Fehlen von Strömungsgeräuschen in normalen Gefässen der Ausdruck des Fehlens von Wirbeln ist. Nur bei gleitender Strömungsart bewegt sich die Flüssigkeit so geräuschlos, wie es im natürlichen Kreislauf der Fall ist.

Und eine weitere Beobachtung führt zu demselben Schluss.

Versebiedenen Tieren (Hund, Katze, Kaninchen) habe ich durch die Karotis eine dünnwandige Glassonde bis zu der Aorta vorgeschoben. Durch die Kanüle liess ich mit mässigem Überdruck eine mit Methylenblau intensiv gefärbte Gummiarabikum-Lösung von der Viskosität 4,5 in die Blutbahn einströmen. Gleichzeitig war in die eine der beiden Kruralarterien eine lange Glaskanüle eingebunden, durch die das ausströmende Blut auf eine Glaskugel übergeleitet wurde. Auf der abfallenden Wölbung fliesst es in einem breiten Stromband auseinander, in dem Blut und Gummilösung zum Teil noch getrennt sichtbar sind. Bêi turbulenter Strömungsform müsste eine vollständige Mischung beider Flüssigkeiten zustande gekommen sein.

Theoretische Grüde also, wie auch direkte Beobachtungen zeigen glejeh deutlich, dass die Fortbewegung des Blutes in den Gefässen in derjenigen Strömungsform geschieht, welche diegeringsten Verluste an Reibung verursacht: es ist dies dieStrömung in parallelen Stromfäden, die „gleitende" Bewegung, für welche das Poiseuille'sche Gesetz gilt.

Damit finden wir auch die dritte unserer Vorraussetzung (vgl. S. 474) in lebenden Gefässsystem erfüllt.: Es steht uns somit kein Grund entgegen, die Resultate unserer mechanischen Analyse für die Kreislaufregulierung auszuwerten.

\section{Die Anwendung der Resultate auf das Blutgefässsystem.}

Durch die Erörterungen in Abschnitt I haben wir erfahren, dass zwischen der Muskulatur, welche entlang denselben Stromfäden liegt, ein synergistisches Verhältnis besteht (vgl. S. 451). Eingangs dieses Abschnittes II haben wir dann die Frage aufgeworfen, ob die Muskulatur der verschiedenen aufeinanderfolgenden Teilstücken mit synergistischer Muskulatur sich im Einfluss auf das Strömen quantitativ äquivalent ist oder nicht.

Wir haben diese Fragestellung erklärt an Hand einer schematischen Skizze, w.ie wir sie in Fig. 2 S. 462 gegeben haben. Bei dem 
skizzierten konkreten Fall zielt die Fragestellung zum Beispiel darauf $\mathrm{ab}$, zu erfahren, in welcher der eingetragenen Zonen $I, I I, I I I$ und $I V$ die Gefässmuskulatur wirksamer den Widerstand zu verändern mag. Wir sind nun in der Lage, auf diese Frage eine präzise Antwort zu geben.

Der Wirkungsgrad der Muskulatur verschiedener aufeinanderfolgender Gefässstrecken hängt davon ab, ob und in welcher Weise die Breite der Strombahn peripherwärts eine Veränderung erfährt. Ist die Strombahnbreite überall dieselbe, so überragt der Einfluss der peripheren Muskulatur bedeutend. Eine peripherwärts $\mathrm{rasch}$ zunehmende Verbreiterung der Strombahn schiebt den Haupteinfluss dagegen der zentralwärts gelegenen Gefässmuskulatur zu. Zwischen diesen beiden Möglichkeiten liegt ein Aufteilungsmodus, wo die Zunahme der Strombahnbreite in einer Proportion erfolgt, welche der Muskulatur von Stamm, von Ästen und Zweigen der Äste dens el ben Wirkungsgrad verleiht. Wo immer wir einen Querschnitt durch die ganze Breite der Strombahn legen, finden wir zu einer gegebenen Änderung des Gesamtquerschnittes die gleiche Änderung des Gesamtwiderstandes. Bei symmetrischer Aufteilung eines Stammes finden wir das Verhältnis, wenn die Summe der Astquersehnitte das 1,26 fache des Stammquerschnittes beträgt. Wir baben den Zustand „identischer regulatorischer Valenzen" der hintereinandẻrgeschalteten Strombahnabschnitte.

Diesen Aufteilungsmodus, einzuhalten, hat für das lebende Gefässsystem ein hervorragendes Interesse; denn er entspricht derjenigen Konfiguration, welche unter sonst gleichen Umständen dem strömenden Blute den kleinstmöglichen Wiảerstand darbietet. Dementsprechend verlangt ein derart gebautes Gefässsystem vom Herzen die kleinstmögliche Druckleistung. Wir seben hierin eine notwendige Ergänzung zu der Durchführung des Ökonomieprinzipes, wie wir sie im Abschnitt $I$ besprochen haben. Dort hat es sich darum gehandelt, vom Herzen die kleinstmögliche Volumleistung zu fordern. Jetzt gilt es, dieses kleinste Sekundenvolumen unter Aufwand des geringsten Widerstandes an seinen Bestimmungort gelangen zu lassen. Wenn auch dies erreicht ist, dann erst herrscht volle Ökonomie in bezug auf die Arbeitsbelastung des Herzens, d. h. soweit sie eine Funktion der Gestaltung des Gefässnetzes ist. Die Bedingungen, unter welchen die Druckökonomie gewährleistet ist, 
haben wir eben kennen gelernt. E's ist kaum zu erwarten, dass das lebende Gefässsystem in seinem Bau wesentliche Abweichungen von dieser ökonomischen Verteilung des Querschnittes auf Stamm und Åste und Zweige der Äste abweicht. Ob diese Erwartung tatsächlich zutrifft, müssen natürlich darauf gerichtete Untersuchungen erst zeigen. Einen günstigen Angriffspunkt für die Prüfung bietet die Kontrolle der Querschnittsverhältnisse bei symmetrischer Zweiteilung, für welche wir die optimale Stromverbreiterung berechnet haben. Untersuchungen hierüber sind, unter Anwendung einer neuen Methode der Querschnittsbestimmung, zurzeit im Gange.

Wenn wir aus Gründen der Analogie geneigt sind, anzuneh̨men, dass der Organismus diese "Zweckmässigkeit" in der Gestaltung der Gefässe anderen Zweckmässigkeiten zur Seite stellt,. zum Beispiel der Anordnung der Knochenspongiosa, so dürfen wir uns nicht verhehlen, dass damit der Kreislaufregulierung eine ebenso hohe als komplizierte Aufgabe zufällt. Die Druckökonomie stellt nämlich die Forderung, dass die optimale Querschnittsverteilung auf Stamm und Äste durch alle Regulationsphasen hindurch aufrechterhalten' bleibt. Dies ist nur möglich, wenn die Querschnittszunahmen bei Regulierungsakten nach einem ganz bestimmten Verhältnis auf Stamm und Aste dosiert sind. Die Beträge, um welche sich die Querschnitte der verschiedenen Gefässabschnitte erweitern, müssen unter sich genau dieselbe Proportion aufweisen wie die Querschnitte der Gefässstrecken selbst. Bei symmetrischer Zweiteilung müssen also die Äste auch das 1,26 fache an Querschnittzuwachs erhalten wie der Stamm. Nur dann besteht $\mathrm{n}$ a $\mathrm{ch}$ der Regulation dasselbe optimale Verhältnis wie vor der Regulation.

Eine derartig auf die verschiedenen Zonen genau dosierte Regulierung hat nun aber seine Schwierigkeit! Wir vermögen sie einzuschätzen, wenn wir uns daran erinnern, dass die Angriffsfront des Gefässmuskels sehr veränderlich ist. Auf keinen Fall würde die differenzierte Querschnittsänderung gewährleistet sein durch ein konstantes Verhältnis der nervösen Impulse an Stamm einerseits und Äste anderseits. Verschiebungen der statischen Druckverbältnisse haben zur Folge, dass ein und dieselbe Spannungsänderung der Gefässmuskulatur das eine Mal einen grösseren, das andere Mal einen kleineren Effekt hat. 


\section{Sensorische Kontrolle der Querschnittsgestaltung der Gefässe.}

Die Lösung der aus Gründen der Druckökonomie überaus wichtigen mechanischen Aufgabe erscheint ungemein kompliziert! Vielleicht ist sie abberhaupt mechanisch unmöglich, wenn der Kreislaufregulierung nicht ein bestimmtes Mittel zur Verfügung steht. Es ist dies ein sensorischer Apparat, welcherdie Querschnittsgestaltung der einzelnen hintereinandergeschalteten Gefässstrecken fortlaufend kontrolliert.

Wenn eine solche sensorische Kontrolle (auf. dem Reflexwege) Einfluss auf den Ablauf der motorischen Akte besitzt; dann sehen wir die Schwierigkeit mit einem Schlage überwunden. Auf welchem Wege, wird uns ein Beispiel zeigen:

Wir betrachten dabei ein Gefässstück, welches wir uns aus drei Abschnitten zusammengesetzt denken, wie dies beispielsweise in Fig. 9 skizziert ist. Die Wandung dieses Gefässes denken wir uns begabt mit einer spezifischen Sensibilität, deren ädaquater Reiz die Wanddehnung ist. Die motorische Antwort auf das Auftreten eines Dehnungsreizes sei eine doppelte, nämlich ein reflektorischer Verengerungsimpuls in Bereiche des gedehnten Abschnittes selbst und ein Erweiterungsimpuls in den anderen Abschnitten.

In diesem Falle steht die Querschnittsgestaltung des $\mathrm{Ab}$ schnittes $I I$ unter dem Einfluss des Dehnungsreizes seiner eigenen Wand. Der Dehnungsreiz strebt eine Entdehnungsreaktion, d. h. erue Verengerung an. Gleichzeitig unterliëgt aber die Querschnittsgestaltung des Abschnittes $I I$ dem Einfluss der Dehnungsreize in den übrigen Abschnitten entlang der Strombahn, also $I$ und $I I I$. Der Einfluss ist ein entgegengesetzter. Es wird eine Reaktion veranlasst im Sinne zunehmender Erweiterung.

So sehen wir im Vèrlaufe einer Erweiterung der ganzen Gefässstrecke den Abschnitt $I I$ unter dem Widerspiel zweier antagonistischer Einflüsse. Übergewicht besitzen die Verengerungsimpulse, wenn die eigene Dehnung grösser ist als die der anderen Abschnitte. Übergewicht besitzen die Erweiterungsimpulse, wenn die eigene Dehnung kleiner ist als die der. rivalisierenden Abschnitte. Gleichgewicht zwischen "Verengerungs- und Erweiterungsimpulsen besteht dann, wenn Dehnungsgleichgewi cht besteht. Mit anderen' Worten: Die Quersehnittsverteilung entlang der Strombahn stabilisiert sich bei diesem Mechanismus in soleher Weise, dass die Wand- 
dehnung aller Teilstücke entlang der Strombahn gleichmässig zunimmt. Wir haben eine automatische Nivellierung auf einen identischen Dehnungszustand der Gefässwände vor uns. Um die Wirkungsweise dieser Nivellierungsprozesse noch etwas leichter verstänảlich zu machen, sei eine Ausdrucksweise gestattet, welche nicht absolut wissenschaftlich klingt, aber die Verhältnisse sehr gut kennzeichnet.

Wir halten dabei eine verzweigte Strombahn im Auge, deren Widerstand aus regulatorischen Gründen herabgesetzt werden soll. Die gesamte Muskulatur der Strombahn erhält einen Erweiterungsimpuls ohne feinere Differenzierung auf die einzelnen Abschnitte. Sowie die Erweiterung effektiv wird, setzt das Antagonistenspiel $z$ wischen den verschiedenen hintereinandergeschalteten Gefässstrecken ein. Durch seine Vermittlung sucht jeder einzelne Abschnitt die vom versorgten Parenchym erzwungene Erweiterung mit entsprechender Wanddehnung von sich abzuwenden und den anderen Abschnitten mit synergistischer Muskulatur zuzuschieben. Anderseits erhält er von diesen ein gewisses Maass von Dehnungszuwachs aufgedrängt. Das sich automatisch einstellende Gleichgewicht ist erreicht im gleichmässigeu Zuwachs der Wanddehnung bei deu einzelnen hintereinandergeordneten Teilstücken, die unter sich um den kleinsten Dehnungszuwachs rivalisieren.

Der Gedanke an eine Sensibilität der Gefässwände ist nicht neu. Die Annahme einer solchen finden wir schon hier und dort angedeutet, wenn auch nicht in bezug auf Qualität und Funktion \$o charakterisiert, wie wir eben ausgeführt ${ }^{1}$ ). Wir werden auf die Versuche und Anschauungen der zitierten Autoren in der nächsten Arbeit zu diesem Thema zu sprechen kommen, wo es gilt, die dargelegte Theorie durch Tatsachen zu erhärten. Nur so viel möge hier schon verwähnt sein, dass nach unserer Vorstellung der Depréssor ein Stück dieses sensorischen Apparates ist, dass wir ferner den Dehnungsreizen in der Funktion der Gefässmuskulatur eine ganz analoge

1) P. Heger, Einige Versuche über die Empfindlichkeit der Gefässe. Beitr. zur Physiol., C. Ludwig gewidmet, S. 193. Leipzig 1887; ferner Spalitta and Consiglio, I Nervi vasosensitivi. Palermo 1896; ferner Delecenne, Compt. rend. t. 124 p. 700.1897 ; ferner Pagano, Arch. jtal. de Biol. t. 33 p. 1. 1900; ferner Siciliano, Arch. ital. de Biol. t. 33 p. 338.1900. 
Rolle zudenken, wie wir sie als sogenannte propriozeptive Reize, von Sehnen und Gelenken ausgehend, bei der Differenzierung und Dosierung der Skelettmuskelfunktion treffen ${ }^{1}$ ).

\section{Theorie zur Regulierung der Blutdruckverteilung.}

In einem früheren Abschnitt haben wir die Theorie der Regulierung der Volumverteilung (Stromvolum) besprochen. Wir lernten einen Mechanismus kennen, welcher eine Teilaufgabe der peripheren Kreislaufregulierung zu erfüllen imstande ist: die nach dem Bedarf gerichtete und gleichzeitig ökonỏmische Verteilung des vom Herzen geförderten Blutvolumens auf die verschiedenen Parenchymbezirke. Wir brauchen nur den Inhalt der letzten Abschnitte zusammenzufassen, um nun auch zu einer konkreten Vorstellung über einen Mechanismus zu gelangen, welcher imstande ist, die zweite Teilaufgabe der peripheren Kreislaufregulierung restlos zu erfüllen, nämlich die ökonomische Verteilung des vom Herzen aufgebrachten Druckes auf die verschiedenen, sich hintereinander anfügenden $\mathrm{Ab}$ schnitte der Gefässbahn.

Infolge der Überwindung von Widerständen beim Strömen des. Blutes nimmt der Blutdruck vom Herzen nach der Peripherie hin ab. In weicher Form diese Abnahme vor sich geht, ob hauptsächlich zu Beginn der Strombahn in den weiten Gefässen mit hoher Strömungsgeschwindigkeit oder aber vorwiegend den en g e n Gefässen mit la ng s a m er Strömung, ist lediglich eine Frage der Querschnittsverteilung entlang der Strombahn. Es kommt darauf an, in welchem Verhältnisse die Querschnitte stehen, die sich entlang denselben Stromfäden aufeinnanderfolgen; es hängt davon ab, wie sich die Breite der Strombahn verändert, wenn sich die einheitliche. Strombahn zum Zwecke der räumlichen Verteilung des Blutes in getrennte Gebiete aufsplittert. Die Fixierung bestimmter Querschnittsbeziehungen zwischen Stamm und Ästen ist gleichbedeutend mit der Fixierung einer bestimmten Druckverteilung entlang der Strombahn, d. h. eines bestimmt verlaufenden Druckgefälles.

Ein Regulierungsmechanismus, welcher die Regulierung jener Querschnittsbeziehungen besorgt, erledigt eo ipso die Regulierung des Druckgefälles.

1) Sherrington, The integrative action of the nervons system. Constable, London 1911. - (Vgl. p. 129 t. ff.: Proprioceptive reflexes.) - v. Monakow, Lokalisàion im Grosshìn. Bergmann, Wiesbaden 1914. (Vgl. S. 173.) 
Über die Funktionsweise dieses zweiten Mechanismus machen wir uns, entsprechend dem Gesagten, folgende Vorstellung:

Die Verteilung der Strömungswiderstände und damit die Regulierung des Druckes entlang der Strombahn (Druckgefälle) basiert auf der Funktion einer spezifischen Dehnungsensibilität der Gefässe.

Dureh die sensorische Erregung wird auf dem Reflexwege eine Rivalität zwischen den einzelnen Gefässstrecken, welche synergistische Muskulatur .tragen, eröffnet, eine Rivalität um den geringeren Dehnungsuwachs (bei regulatorischer Erweiterung). Das Resultat dieser Rivalität ist eine gleichmässige Verteilung der Dehnungszunahme; welche mit jeder Gefässerweiterung bzw. Widerstandsverminderung notwendigerweise verbunden ist.

Eine dermaassen geleitete regulatorische Erweiterung von Gefässen lässt die Bedingungen in Erfüllung gehen, dass die Gefässmuskelakte ihren regulatorischen Zweck mit der kleinstınöglichen Querschnittserweiterung erreichen. Ein Erfolg solcher Art Regulierung ist es ferner, dass ein aus engsten Gefässbahnen sich herausbildendes Gefässsystem sich automatisch auf diejenige Qüerschnittsverteilung einstellt, die einen bestimmten Widerstand mit dem kleinstmöglichen Querschnitt verbindet (vgl. S. 471). Zu diesem Ziele hin wird die Entwicklung der Strombahn dann geleitet, wenn sich die beschriebene Rivalität zwischen einzelnen Gefässabschnitten nicht nur bei Regulierungen auf temporären Mehrbedarf des Gewebes betätigt, sondern auch auf dauernden Mebrbedarf, wie es durch Wachstum der Organe bedingt ist.

Mit diesen Ausführungen über die Regulierung der Druckverteilung haben wir unsere im Abschnitt I entwickelte Vorstellung in der Weise ergänzt, dass wir ein abgeschlossenes Bild der Funktionsweise der peripheren Kreislaufregulierung gezeichnet haben. Wenn wir in der dargelegten Theorie eine Auswertung unserer analytischen Resultate suchen, so geschieht es ausdrücklich nicht in der Absicht, theoretische Deduktionen an Stelle experimenteller Untersuchungen treten zu lassen, sondern $\mathrm{konkrete}$ Angriffspunkte zu schaffen für eine folgende experimentelle Behandlung des Problems, wobei wir nun von der Dynamik derGefässmuskulatur als Grundlage ausgehen. Bei einem solchen Vorgehen, das sich entgegen bisheriger Übung nich t über eine genauere Kenntnis der für den Mechanismus der Kreislaufregulierung maassgebenden mechanischen Prinzipien" hinwegsetzt, erwarten wir nicht 
nur eine nützliche Wegleitung für die folgenden experimentellen Untersuchungen, sondern auch für das bessere Verständnis mancher beute schon bekannter Erscheinungen.

\section{Die Regulierung von Tolum- und Druckverteilung in vereinigter Funktion.}

Eine funktionstüchtige Kreislaufregulierung hat zwei vollständig differenten Aufgaben gerecht zu werden:

1. die Regulierung des Stromvolumens, bei dessen Verteilung an die einzelnen Parenchymbezirke;

2. Regulierung des Druckgefälles entlang der Strombahn.

Die beiden Funktionen werden durch zwei verschiedenartige Mechanismen erledigt.

\section{Ad 1. Die Regulierung des Stromvolumens.}

Die Volumregulierung wird beherrscht von einer spezifischen Durchblutungssensibilität im Parenchym, deren Erregung die den Blutstrom umsteuernden Gefässmuskelakte auslöst und dosiert.

Das mechanische Mittel der Volumregulierung ist die Fixierung bestimmter Querschnittsverhältnisse zwischen parallelgeschalteten Abschnitten des Gefässnetzes (Strombezirken).

Es wird durch innervatorische Beziehungen zwischen konkurrierenden Gewebe- und Gefässbezirken in Wirksamkeit gesetzt. Die motorischen Akte, welche dabei zustande kommen, bestehen in einer assoziierten Betätigung von Agisten und Antagonisten. Die Konsequenz dieser Assoziation ist eine Rivalität zwischen den verschiedenen parallelgeschalteten Strombezirken um das vom gemeinschaftlichen Stamme zugeführte Blut. Der Erfolg dieser Rivalität ist eine derartige Aufteilung der vom Zentrum gelieferten Gesamtblutmenge, welche eine ausreichende Durchblutung sämtlicher Gewebe mit der kleinstmöglichen Gesamtblutmenge ermöglicht.

\section{Ad 2. Regulierung des Druckgefälles.}

Die Regulierung des Druckgefälles wird beherrscht von einer Sensibilität in den Gefässwänden, deren Erregung die Gefässmuskelaktion auslöst, welche die Druckverteilung entlang der Strombahn verschiebt.

Das mechanische Mittel der Regulierung des Druckgefälles ist die Fixierung bestimmter Querschnittsverhältnisse zwischen hinter- 
einander geschalteten Abschnitten des Gefässnetzes (Zonen der Strombahnen).

Es wird in Wirksamkeit gesetzt durch innervatorische Beziehungen zwischen den einzelnen aufeinanderfolgenden Gefässstrecken. Die motorischen Akte, welche dabei zustande kommen, entstehen unter dem Einfluss von Verengerungsimpulsen am Ort der Dehnung ' ausgelöst und Erweiterungsimpulsen, die von "den synergistischen Internodien zugeleitet werden.

Die Konsequenz dieser doppelten Innervation ist Rivalität zwischen den verschiedenen synergistische. Muskulatur tragenden Gefässstrecken, eine Rivalität, deren Erfolg eine solche Querschnittsverteilung zwischen Stamm und Ästen und Zweigen der Äste ist, die den Strömungsablauf mit kleinstmöglichem Druckgefälle gewährleistet. Durch die Vereinigung der beiden Mechanismen, welche einerseits die Querschnittsverbältnisse zwischen parallel geschalteten, andererseits zwischen hintereiuander geschalteten Gefässstrecken regeln, sehen wir die Aufgabe der peripheren Kreislaufregulierung restlos gelöst und gleichzeitig unter die Herrschaft des Ökonomieprinzipes gestellt!

Die Gegenüberstellung von der Regulierung des Stromvolumens und Regulierung des Druckgefälles sei noch durch die schematischen Skizzen ergänzt, wie sie Fig. 12 a und 12 b darstellen.

Die erste gibt das Schema für die Regulierung der Volumverteilung. Die Kreise bedeuten zwei getrennte Versorgungsgebiete, in der sich die Stromfäden je eines Astes aufteilen (angedeutet durch die ausgezogenen Pfeile).

Aus jedem Strombezirk herius gehen Frweiterungsimpulse (punktierte Pfeile mit + -Zeichen) zum eigenen Versorgungsgefäss, gleichzeitige Verengerungsimpulse zum Versorgungsgefäss des Konkurenzgebietes (ausgezogene Pfeile mit - Zeichen). An jedem der beiden Gefässe entspinnt sich somit die Rivalität żweier antagonistischer Impulse $(+-)$. Die Frage, ob Gefässnervenzentren in jedem Falle eine vermittelnde Rolle spielen, sei hier nicht berübrt.

Fig. $12 \mathrm{~b}$ ist das Schema für die Regulierung des Druckgefälles: Von der Wandung eines jeden Internodiums nehmen sowohl Verengerungsimpulse (gestrichelte Pfeile mit - Zeichen) und Erweiterungsimpulse (punktierte Pfeile mit + -Zeichen) ihren Ursprung. Erstere gehen an die Muskulatur des Ursprungortes zurück, letztere an die synergistischen Internodien. 
Infolge der wechselseitigen Beziehungen zwischen den verschiedenen Internodien entwickelt sich an jedem einzelnen Internodium die Rivalität antagonistischer Impulse (+ -).

(Wieder bleibt die Frage nach Art nervöser Verbindung unberührt.)

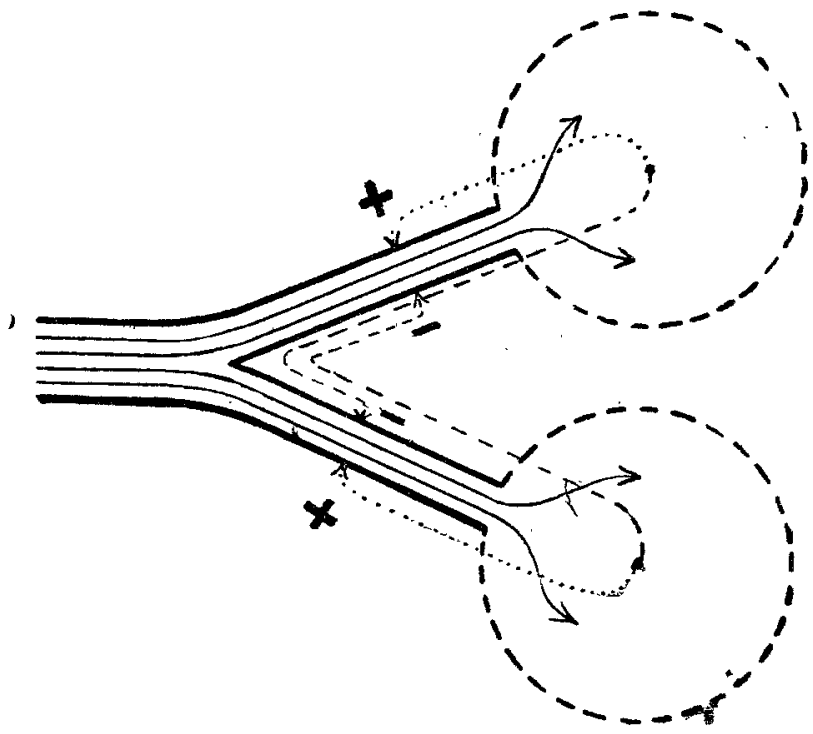

Fig. 12 a.

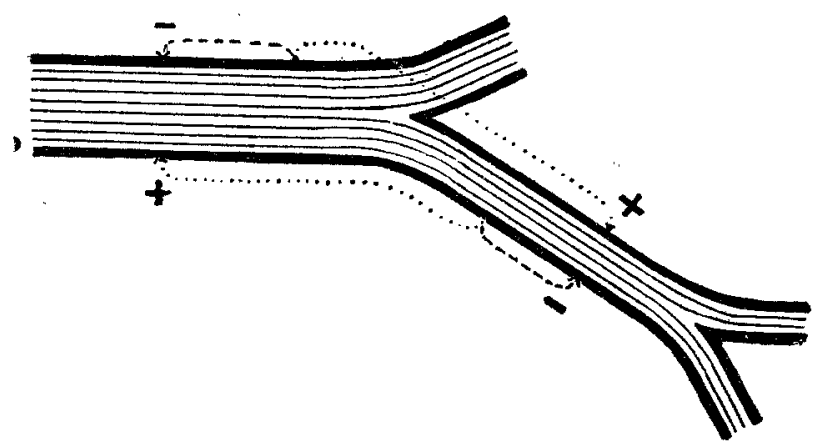

Fig. $12 \mathrm{~b}$.

Mit den eben geschlossenen Ausführungen haben wir die Angriffspunkte für eine hier einsetzende experimentelle Behandlung der Frage gefunden. Als erstes werden wir nun zu entscheiden versuchen, ob die periphere Kreislaufregulierung tatsächlich über jene spezifischen Sensibilitäten verfügt, deren Existenz wir aus den dargelegten dynamischen Gründen für eine zweckentsprechende Betätigung des̉ Gefässmuskelapparates als unentbehrlich erachten. 


\section{Anhang.}

1. Die Beziehung zwischen Quersehnitt und Widerstand einer ungeteilten Strombahn, welche durch ein einzelnes Rohr dargestellt wird.

Der Widerstand einer Strombahn für Flüssigkeiten sei definiert durch die Gleichung

$$
V_{s}=\frac{P}{W} \quad \text { • . . . . . . . }
$$

wobei $V_{s}$ das Sekundenvolumen, $P$ die Druckdifferenz zwischen Anfang und Ende der Strombahn und $W$ der Widerstand bedeutet.

Die Faktoren, welche das Sekundenvolumen bestimmen, sind festgelegt in der Pois euille'schen Formel:

$$
V_{s}=\frac{\pi}{8 \eta \bar{l}} P q^{2} . . . . . . .
$$

$l$ bedeutet die Länge der Strombahn,

$\eta$ die Viskosität der strömenden Flüssigkeit,

$q$ den kreisförmigen Querschnitt der die Strombahn bildenden Röhre.

Aus der Formel 1 und 2 ergibt sich

$$
\frac{P}{W}=\frac{\pi}{8 \eta l} P q^{2}, \quad W=\frac{8 \eta l}{\pi} \cdot \frac{1}{q^{2}} .
$$

Für die Verhältnisse, für welche die Po is e uịlle'sche Formel , von uns hier interpretiert wird, ist $q$ die einzige Variable; die Länge der Strombahn sowie die Viskosität der Flüssigkeiten sind konstant. Wir fassen diese Grössen mit den anderen Konstanten im Faktor $K$ zusammen. Als Beziehung zwischen Querschnitt und Widerstand ergibt sich somit für eine einfache röhrenförmige Strombahn mit kreisförmigem Quersehnitt:

$$
W=\frac{K}{q^{2}} \cdot \cdot \cdot \cdot \cdot \cdot \cdot \cdot .
$$

2. Beziehung zwischen Widerstand und Querschnitt einer Strombahn, welche durch zwei parallelgeschaltete gleiche Röhren dargestellt wird.

Unter der Voraussetzung, dass der Druck je zu Anfang und zu Ende der Strombahn in beiden Röhren ein übereinstimmender ist, lässt sich der Widerstand der aus zwei Röhren dargestellten Strom- 
bahn aus den Einzelwiderständen der beiden Röhren nach der Formel berechnen:

$$
\frac{1}{W}=\frac{1}{W_{1}}+\frac{1}{W_{2}} \text {. . . . . . . . }
$$

wobei $W_{1}$ der Widerstand der einen Röhre, $W_{2}$ der Widerstand der parallelgeschalteten zweiten Röhre ist. $W$ ist der Widerstand der beiden zu gemeinschaftlicher Strombahn vereinigten Röhren.

Nach Gleichung 3 ist:

$$
\begin{aligned}
& W_{1}=\frac{K}{q_{1}^{2}}, \text { daraus folgt } \begin{array}{c}
1 \\
W_{1}
\end{array}=\frac{q_{1}^{2}}{K}, \\
& W_{2}=\frac{K}{q_{2}{ }^{2}}, \text { daraus folgt } \frac{1}{W_{2}}=\frac{q_{2}{ }^{2}}{K} .
\end{aligned}
$$

Für $\stackrel{1}{\underset{W}{W}}=\frac{1}{W_{1}}+\underset{W_{2}}{1}$ ergibt sich unter Benutzung von Gleichung 4 :

$$
\frac{1}{W}=\frac{q_{1}^{2}+q_{2}^{2}}{K}
$$

oder

$$
W=\underset{q_{1}^{2}+q_{2}^{2}}{K} \cdot \ldots \cdot \text {. . . }
$$

Wir setzen nun voraus, dass beide Röhren gleichen Querschnitt baben.

$$
q_{1} \cdots \dot{q}_{2}, \text { damit wird } W=\frac{K}{2} \frac{\frac{K}{q_{1}^{2}}}{} .
$$

Zähler und Nenner mit 2 multipliziert ergibt $W=\frac{2 K}{\left(2 q_{1}\right)^{2}}$.

$2 q_{1}$ ist (weil $q_{1}=q_{2}$ ) die Querschnittssumme beider parallelgeschalteten Röhren. Wir bezeichnen diese Summe mit $q_{n}=2 q_{1}=q_{1}+q_{2}$.

Die Beziehung zwischen Widerstand und Querschnittssumme zweier parallelgeschalteter gleich weiter Röhren ist gegeben in der Gleichung:

$$
W=\begin{gathered}
2 K \\
q_{n}^{2}
\end{gathered}
$$

3. Bestimmung des Querschnittsverhältnisses zweier hintereinandergeschalteter Strecken einer ungeteilten Strombahn, bei denen .der Differentialquotient von Widerstand nach Querschnitt gleich gross ist.

Der Querschnitt der ersten Strecke betrage $q_{n}$ und ihr Widerstand $W_{u}$. Der Querschnitt einer anderen Strecke betrage $q_{b}$ und ihr Widerstand $W_{\iota}$. 
Laut Voraussetzung sei

$$
\frac{d W_{a}}{d q_{a}}=\frac{d W_{b}}{d q_{b}}
$$

Unter Benutzung der Gleichung 3 ergibt sich hieraus

$$
\frac{d}{d q_{a}}\left(\frac{K}{\dot{q}_{a}^{2}}\right)=\frac{d}{d q_{b}}\left(\frac{K}{q_{b}{ }^{2}}\right), \quad \frac{-2 K}{q_{a}{ }^{3}}=\frac{-2 K}{q_{b}{ }^{3}}, \quad q_{a}=q_{b} \text {. }
$$

Resultat: Zwei hintereinandergeschaltete Strecken einen ungeteilten Strombahn mit gleichem Differentialquotient von Widerstand nach Querschnitt sind unter sich querschnittsgleich.

4. Restimmung des Verhältnisses von Stammquersehnitt zur Summe der Astquerschnitte bei Gleichheit des Differentialquotienten in der Stamm- und der Astzone.

Gleichheit der Differentialquotienten besteht, wenn

$$
\begin{aligned}
\frac{d}{d q_{a}}\left(\frac{K}{q_{a}{ }^{2}}\right) & =\frac{d}{d q_{n}}\left(\frac{2 K}{q_{n}^{2}}\right) \text { (aus Gleichung } 3 \text { und } 6 \text { ), } \\
\frac{-2 K}{q_{u}^{3}} & =\frac{-2 \cdot 2 K}{q_{n}^{3}}, \quad q_{n}{ }^{3}=\frac{q_{n}{ }^{3}}{2}, \quad q_{n}=\sqrt[3]{2} q_{n} .
\end{aligned}
$$

Resultat: Für eine zusammengesetzte Strombahn mit $q_{a}$ als Stammquerschnitt, mit $q_{n}$ als Querschnittssumme zweier gleicher Äste besteht Gleichheit des Differentialquotienten, wenn die Summe der Astquerscbnitte das 1,26 fache $(=\sqrt[3]{2})$ des Stammquerschnittes beträgt.

5. Gleichheit des Differentialquotienten von Widerstand nach Querschnitt bei allen aufeinanderfolgenden Strombahnabschnitten ist: das Merkmal einer Strombahn mit kleinstmöglichem Widerstand.

Beweis: Eine Strombahn von gegebener Länge aber beliebiger Querschnittskonfiguration soll ohne Veränderung der Länge und ohne Veränderung des mittleren Querschnittes (d. h. also auch bei gleichbleibendem Gesamtinhalt) derart eine Querschnittsverschiebungerfahren, dass der Gesamtwiderstand verringert wird.

Die .Erreichung dieses Zieles setzt voraus, dass irgendwo im System ein Abschnitt existiert, dessen Erweiterung den Widerstand stärker herabsetzt, als ị̣n eine gleich starke Verengerung irgendeines anderen gleich langen Abschnittes erhöht. Fine solche Stelle existiert nicht, sobald der Differentialquotient von Widerstand nach Querschnitt bei allen aufeinanderfolgenden Strombahnabschnitten 
490 W. R. Hess: Über die periphere Regulierung der Blutzirkulation.

der gleiche ist. Unter diesen Bedingungen besteht also keine Möglichkeit, bei der gegebenen Strombahn eine andere Querschnittskonfiguration herbeizuführen, welche durch einen kleineren Widerstand ausgezeichnet ist. Das Widerstandsminimum ist bereits erreicht.

6. Reyno I d'sche Formel ${ }^{1}$ ): $\frac{v s d}{\eta}$.

$v=$ mittlere Geschwindigkeit,

$s=$ spezifisches Gewicht der strömenden Flüssigkeit,

$d=$ Durchmesser der Röhre,

$\eta=$ Koeffizient der inneren Reibung.

1) Aus Kohlrausch, Lehrb. d. prakt. Physik, 11. Auf., S. 259. 